\title{
A genome-wide RNAi screen identifies genes regulating the formation of $P$ bodies in C. elegans and their functions in NMD and RNAi
}

\author{
Yinyan Sun ${ }^{1 *}$, Peiguo Yang ${ }^{1,2 *}$, Yuxia Zhang ${ }^{1 *}, X_{i n ~ B a o}{ }^{1}$, Jun $\mathrm{Li}^{1}$, Wenru Hou ${ }^{1}$, Xiangyu Yao $^{1}$, Jinghua Han ${ }^{1}$, \\ Hong Zhang ${ }^{1 \oplus}$ \\ ${ }^{1}$ National Institute of Biological Sciences, Beijing 102206, China \\ 2 College of Life Sciences, Beijing Normal University, Beijing 100875, China \\ $\triangle$ Correspondence: zhanghong@nibs.ac.cn \\ Received October 9, 2011 Accepted October 18, 2011
}

\section{ABSTRACT}

Cytoplasmic processing bodies, termed $\mathbf{P}$ bodies, are involved in diverse post-transcriptional processes including mRNA decay, nonsense-mediated RNA decay (NMD), RNAi, miRNA-mediated translational repression and storage of translationally silenced mRNAs. Regulation of the formation of $P$ bodies in the context of multicellular organisms is poorly understood. Here we describe a systematic RNAi screen in $C$. elegans that identified 224 genes with diverse cellular functions whose inactivations result in a dramatic increase in the number of $P$ bodies. 83 of these genes form a complex functional interaction network regulating NMD. We demonstrate that NMD interfaces with many cellular processes including translation, ubiquitin-mediated protein degradation, intracellular trafficking and cytoskeleton structure. We also uncover an extensive link between translation and RNAi, with different steps in protein synthesis appearing to have distinct effects on RNAi efficiency. Moreover, the intracellular vesicular trafficking network plays an important role in the regulation of RNAi. A subset of genes enhancing $P$ body formation also regulate the formation of stress granules in C. elegans. Our study offers insights into the cellular mechanisms that regulate the formation of $P$ bodies and also provides a framework for system-level understanding of NMD and RNAi in the context of the development of multicellular organisms.
KEYWORDS $P$ body, stress granules, nonsensemediated RNA decay (NMD), RNA interference, C. elegans

\section{INTRODUCTION}

Distinct classes of ribonucleoprotein (RNP) granules appear to function in specific aspects of RNA metabolism (Anderson and Kedersha, 2006). Cytoplasmic processing bodies, termed $\mathrm{P}$ bodies, are involved in mRNA degradation, nonsense-mediated RNA decay (NMD), siRNA- and microRNA (miRNA)-mediated gene silencing in mammalian cells (Sheth and Parker, 2003; Cougot et al., 2004; Jakymiw et al., 2005; Liu et al., 2005a, 2005b; Sen and Blau, 2005; Bruno and Wilkinson, 2006; Parker and Sheth, 2007). Consistently, $P$ bodies contain components involved in $5^{\prime}$ to $3^{\prime}$ mRNA degradation, including the decapping complex DCAP1/ DCAP2, decapping coactivators (e.g. RCK/p54, LSM1-7 complex, EDC3, Hedls and elF4E-T), the CCR4/NOT1 deadenylase complex and the exonuclease XRN1 (Parker and Sheth, 2007). Components required for NMD (e.g. SMG5, SMG7, UPF1), siRNA- and miRNA-mediated gene silencing (e.g. AGO, GW182 and RAP55) are also localized in $P$ bodies (Parker and Sheth, 2007). The formation of $P$ bodies is also regulated by components involved in these RNA metabolism processes (Parker and Sheth, 2007). Loss of function of components involved in the $5^{\prime}$ to $3^{\prime}$ mRNA degradation process prior to decapping, including elF-4E-T, LSM1, RCK/p54 and CCR4, leads to reduction or disappearance of $\mathrm{P}$ bodies, while disruption at or after decapping,

\footnotetext{
"These authors contributed equally to the work.
} 
including decapping complex component DCAP2 and subsequent $5^{\prime}$ to $3^{\prime}$ mRNA decay XRN1, results in accumulation of early components in P bodies. Blocking RNAi and miRNA silencing pathway prevents the $P$ body formation. In the physiologically intact animal, little is known about the mechanisms governing the assembly of $\mathrm{P}$ bodies and other RNP granules.

Several other ribonucleoprotein granules in mammalian cells, including stress granules (SGs) and neuronal granules, are biochemically and functionally related to $\mathrm{P}$ bodies (Anderson and Kedersha, 2006). SGs are physically distinct and spatially separable from $\mathrm{P}$ bodies, playing a role in storage of translationally repressed mRNAs under stress conditions (Kimball et al., 2003; Kedersha et al., 2005). The assembly of SGs appears to be regulated by distinct signaling pathways in mammalian cells. The assembly of SGs, but not $P$ bodies, requires the phosphorylation of translation initiation factor elF2, which can be induced by various stresses, including heat shock and ER stress (Kedersha et al., 1999; Anderson and Kedersha, 2006). In yeast, where distinct SGs are not present, $\mathrm{P}$ bodies are involved in mRNA decay and NMD and also assume the SG function of storage of translationally repressed mRNAs (Brengues et al., 2005). The assembly of $P$ bodies in yeast displays many common properties with the formation of SGs, such as the induction of SG formation by phosphorylation of elF-2 (Anderson and Kedersha, 2006). Thus, the formation of $P$ bodies appears to be controlled by distinct mechanisms among different systems.

NMD degrades aberrant transcripts containing a premature termination codon (PTC), preventing possible deleterious effects of truncated proteins (Maquat, 2004). The mechanisms underlying the recognition of PTC vary among organisms. In mammalian cells, the exon junction complex (EJC), including Y14, Magoh, elF4AIII and Barentsz, is required for recognizing PTC and triggering NMD (Isken and Maquat, 2007). In C. elegans and Drosophila, PTC recognition occurs independent of introns and consequently, component of EJC is dispensable for NMD (Mango, 2001; Longman et al., 2007). However, the core NMD machinery components, including UPF1, UPF2, UPF3, SMG1, SMG5 and SMG7, are evolutionarily conserved (Behm-Ansmant and Izaurralde, 2006; Isken and Maquat, 2007). In C. elegans, the involvement of smg-1 to -7 in NMD was uncovered due to their roles in allelespecific but gene-nonspecific suppression of a PTC containing mutation affecting a variety of genes (Hodgkin et al., 1989). Essential genes, however, have not been isolated in previous genetic screens, as suppression of the relevant phenotypes could only be scored at post-embryonic stages and only viable and relatively healthy mutants were isolated. The molecular mechanisms regulating NMD and the role of $P$ bodies in NMD remain largely unknown in multicellular organisms.

Here we describe a genome-wide RNAi screen in
C. elegans that identified 224 genes whose inactivations resulted in an increase in the number of $\mathrm{P}$ bodies. We further examined the role of the identified genes in NMD and RNAi. Our study revealed important differences in the regulation of $P$ body formation between $C$. elegans and yeast or mammalian cells and also showed that a subset of genes regulating the formation of $\mathrm{P}$ bodies also play an important role in NMD and RNAi.

\section{RESULTS}

\section{Formation of $P$ bodies is temporally regulated in C. elegans}

A translational reporter for the $\mathrm{P}$ body-specific marker dcap$1:: r f p$, which encodes the $C$. elegans ortholog of decapping complex component DCAP1, was constructed to examine the formation of $P$ bodies in developing animals. This reporter is functional in rescuing developmental defects associated with the dcap-1(tm3163) mutation, including weak uncoordinated (Unc) and small brood size phenotypes (Fig. 1A). dcap-1:::rfp was weakly expressed and homogenously distributed in the cytoplasm at all stages of embryogenesis (Fig. 1B and 1C). After hatching, DCAP-1::RFP also aggregated into granules, especially in the head and tail regions (Fig. 1D and 1E). The number and size of aggregates were gradually increased as the animal grew (Fig. 1F-1K). Many big DCAP-1::RFP aggregates were formed throughout the body in old adult animals (Fig. $1 \mathrm{~L}$ and $1 \mathrm{M}$ ). Thus, the formation of $\mathrm{P}$ bodies appears to be temporally regulated and is correlated with developmental timing.

\section{Formation of $\mathrm{P}$ bodies in NMD, RNAi and miRNA pathway mutants}

We next examined the formation of $\mathrm{P}$ bodies in mutants with defects in various RNA metabolism processes. Mutations in dcap-2, encoding the catalytic subunit of the decapping complex, resulted in a dramatic increase in the formation of DCAP-1::RFP bodies at post-embryonic stages (Fig. 2A and 2B). The mRNA level of dcap-1, however, was not upregulated in dcap-2 mutants (Fig. $2 \mathrm{C}$ ), suggesting that enhanced formation of $\mathrm{P}$ bodies in dcap-2 mutants is posttranscriptionally regulated, such as aggregation of diffuse DCAP-1 proteins into bodies. The formation of $P$ bodies was also enhanced by RNAi inactivation of other components involved in the $5^{\prime}$ to $3^{\prime}$ mRNA decay at a step after decapping, such as $x r n-1$ and $x r n-2$ (Fig. 2D). RNAi inactivation of components that function in the $5^{\prime}$ to $3^{\prime}$ mRNA decay at a step prior to decapping, such as $I s m-1,-2$, or -3 , weakly reduced the formation of DCAP-1 bodies (Fig. 2E).

To examine whether impaired NMD has an effect on the formation of $\mathrm{P}$ bodies, the expression of dcap-1::rfp was examined in smg-1, -2, -4 , and -5 RNAi animals and no 

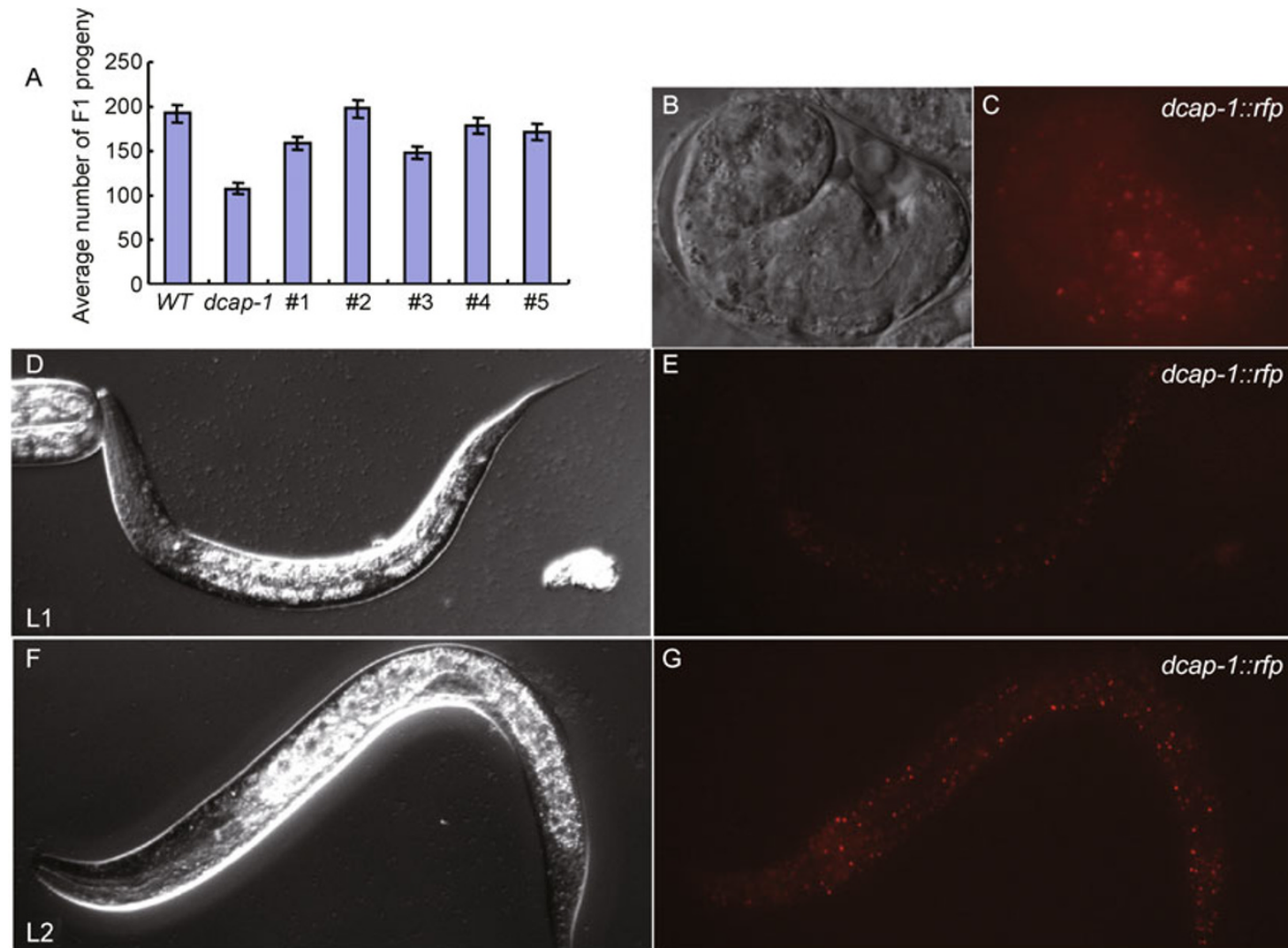

G

dcap-1::rfp
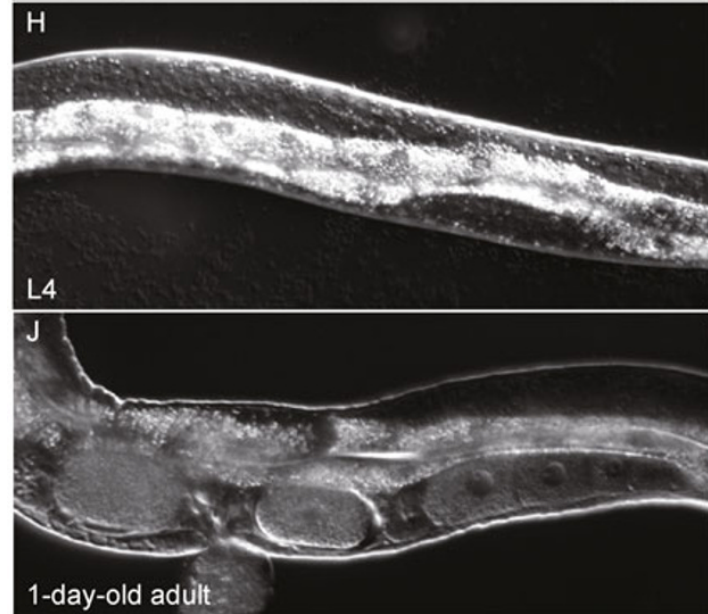

K

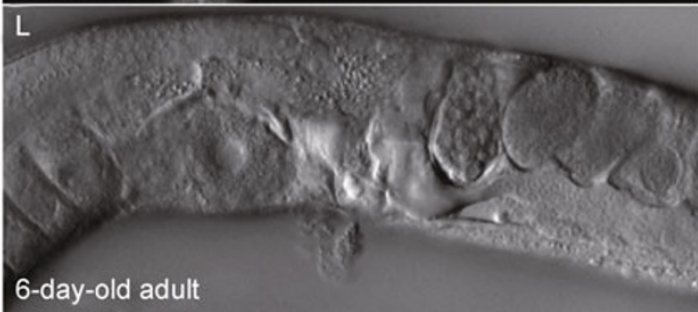

M

dcap-1::rfp

Figure 1. Expression pattern of dcap-1::rfp. (A) The developmental defects in dcap-1(tm3163) mutants are rescued in five separate lines carrying the $d c a p-1:$ : ffp transgene (labeled as \#1 to \#5). The average number of F1 progeny was derived from 10 hermaphrodites in each line. (B and C) dcap-1:: ffp is weakly expressed in embryos. (B) Nomarski image of the embryo shown in (C). (D-M) At post-embryonic stage, DCAP-1::RFP forms aggregates and the number and size of aggregates increase as development proceeds. (D and E) L1 stage; ( $F$ and $G) L 2$ stage; ( $H$ and I) $L 4$ stage; ( $($ and $K)$ 1-day-old adult; ( $L$ and $M)$ 6-day-old adult. (D), ( $F$ ), $(H),(J)$ and $(L)$ Nomarski images of the animals shown in $(E),(G),(I),(K)$ and $(M)$, respectively. 

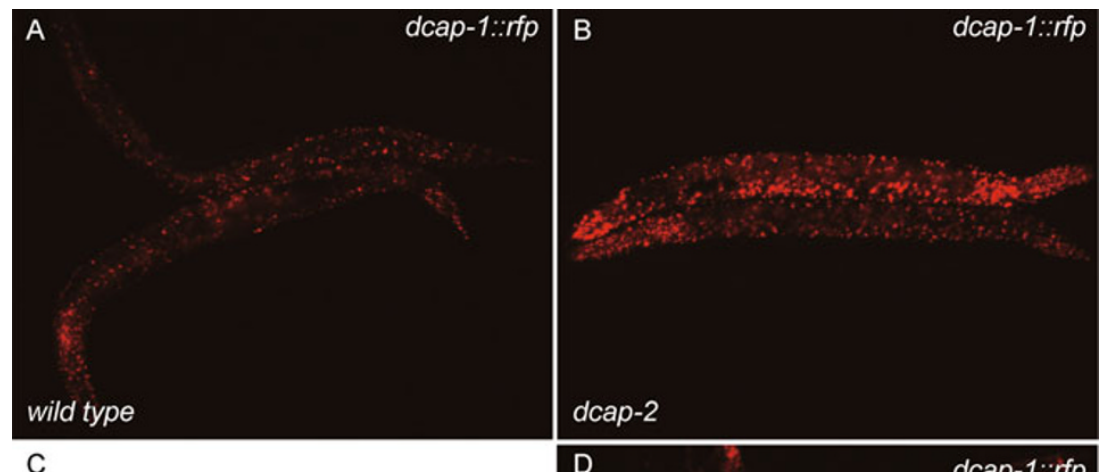

C
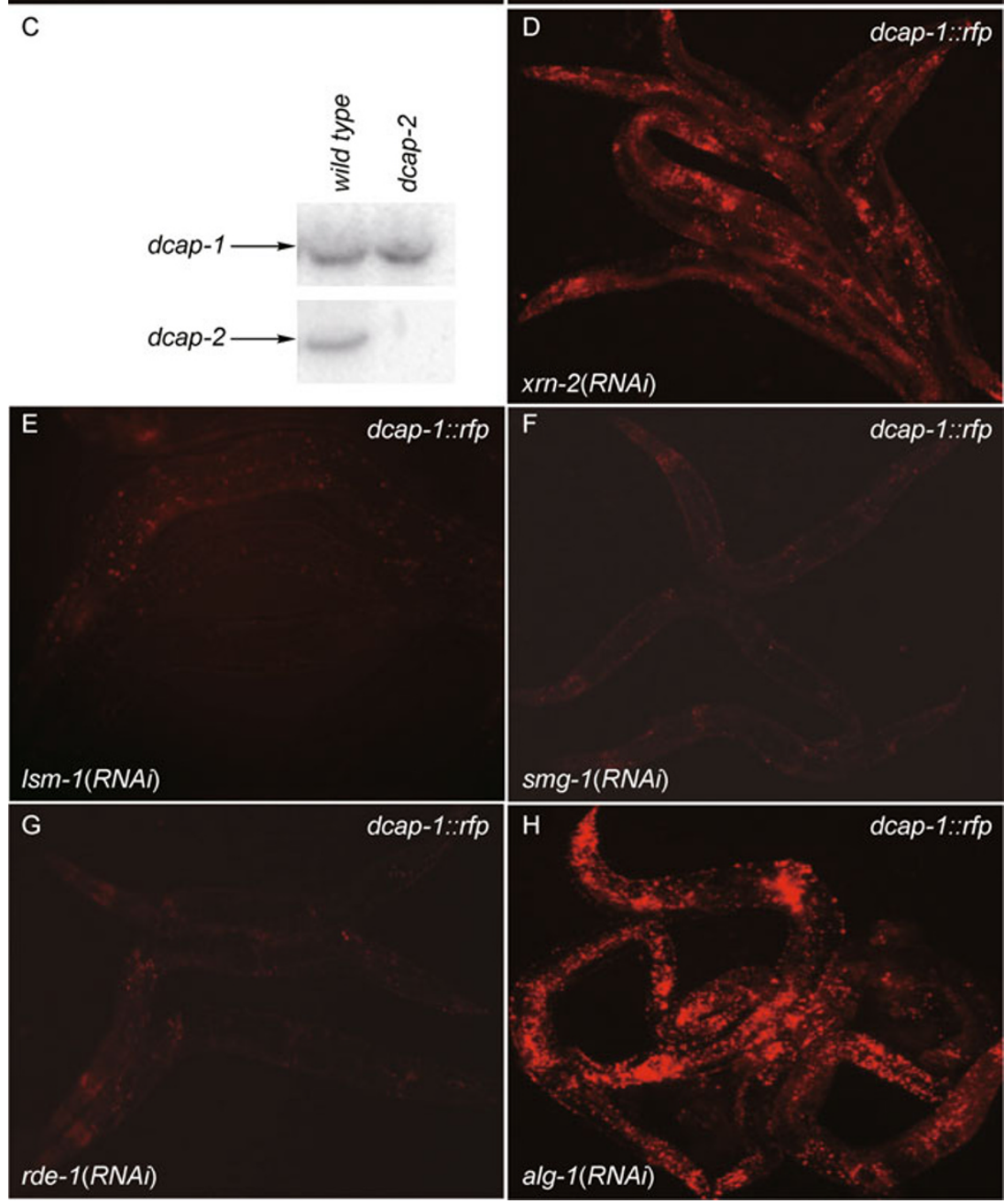

Figure 2. Expression of dcap-1::rfp in various genetic backgrounds. (A) Formation of DCAP-1::RFP positive $\mathrm{P}$ bodies in a wild type animal. (B) Enhanced formation of $\mathrm{P}$ bodies in dcap-2 mutants. (C) The mRNA level of dcap-1, detected by Northern blot, was not affected in dcap-2(ok2023) mutants. (D) Weakly enhanced formation of $\mathrm{P}$ bodies in xrn-2(RNAi) animals. (E) Weakly reduced formation of $\mathrm{P}$ bodies in $I s m-1(R N A i)$ animals. Ism-1(RNAi) animals are arrested at larval stages. (F) RNAi inactivation of $s m g-1$ has no effect on the formation of $P$ bodies. (G) RNAi inactivation of $r d e-1$ has no effect on the formation of $P$ bodies. (H) Enhanced formation of $\mathrm{P}$ bodies in alg-1(RNAi) animals. 
obvious changes could be observed (Fig. 2F). We further determined the role of components required for RNAi in regulating the formation of $\mathrm{P}$ bodies and found that RNAi inactivation of factors required for RNAi, including $r d e-1, r d e-$ 4, dcr-1, mut-7 and mut-16, did not change the formation of $P$ bodies (Fig. 2G). Inactivation of alg-1 (encoding the Argonaute homolog) and ain-1 (encoding the GW182 homolog), which are specifically involved in the miRNA pathway, resulted in the accumulation of $\mathrm{P}$ bodies (Fig. $2 \mathrm{H}$ ). Blocking any step in RNAi and miRNA mediated gene silencing in mammalian cells prevents the formation of $P$ bodies. Thus, RNAi and miRNA pathways have a distinct role in regulating the formation of $\mathrm{P}$ bodies in $\mathrm{C}$. elegans and mammalian cells.

\section{$\mathbf{P}$ bodies are colocalized with stress granules in C. elegans}

To investigate whether SGs are present in C. elegans, we examined the expression of a translational reporter for tia-1, which encodes the $C$. elegans homolog of the mammalian SG-specific component TIA-1. TIA-1::GFP was homogenously distributed in the cytoplasm during embryogenesis. At larval stage, strong diffuse TIA-1::GFP signals were observed in seam cells and hypodermal cells (Fig. 3A and 3B). TIA-1::GFP formed distinct bodies, especially in seam cells and hypodermal cells, under stress conditions (e.g. heat shock) (Fig. 3C). Thus, as in mammalian cells, C. elegans TIA-1 forms aggregates in response to stress.

To determine whether SG and P bodies are separable in C. elegans, animals carrying both the tia-1::gfp and dcap-1:: rfp reporters were examined. We found that $68 \%$ of TIA-1 bodies $(n=360)$ were colocalized with P bodies (Fig. 3C-3I). Granules containing only TIA-1::GFP $(24 \%, n=360)$ or DCAP-1::RFP signal $(8 \%, n=360)$ were also present (Fig. 3C-3I). Therefore, unlike in mammalian cells, in which SG and $P$ bodies are spatially separable (Anderson and Kedersha, 2006), TIA-1 and P bodies are mainly colocalized in C. elegans.

\section{Genome wide screen for RNAi clones with increased numbers of $P$ bodies}

To investigate how the formation of $P$ bodies is regulated in $C$. elegans, we screened an RNAi feeding library that individually inactivates 16,644 genes (targeting $\sim 87 \%$ of $C$. elegans ORFs) for mutants with abnormal formation of $P$ bodies, including changes in $\mathrm{P}$ body number or size. To increase the RNAi efficiency, the strain used for screen also carried a mutation in rrf-3, which renders worms to be hypersensitive to RNAi (Simmer et al., 2002). The expression level of dcap-1:: rfp was not obviously affected by an rrf-3 mutation. L1 larvae were seeded and late larvae (for those arrested at larval or adult stages) or F1 progeny was examined for expression of dcap-1::rfp. The RNAi clones that significantly enhanced the formation of $\mathrm{P}$ bodies, compared to the one in wild type animals, were scored positive. RNAi clones identified in the first round of screening were tested three more times. 360 RNAi clones that enhanced the formation of $P$ bodies in at least three tests were selected for further analysis.

To determine whether the identified RNAi clones nonselectively elevated the expression of a transgene, we examined the role of these RNAi clones in the expression of a ubiquitously expressed reporter let-858p::gfp and found that 136 RNAi clones led to the increased expression level of let858p::gfp. 224 RNAi clones that specifically enhanced the expression of dcap-1::rfp were further characterized in this study (Table 1 and Table S1). Of the 224 RNAi clones, 182 gene knockdowns caused larval arrest or sterility. The formation of $P$ bodies was not obviously altered by RNAi inactivation of the remaining $\sim 1300$ essential genes, indicating the specificity of the identified genes in regulating the formation of $\mathrm{P}$ bodies.

Among 224 candidate genes, RNAi inactivation of 80 genes caused a more pronounced increase in $\mathrm{P}$ body number in specific tissues, such as embryos, the germline and the intestine (Fig. 4A-4F). We also identified 8 genes whose inactivations enhanced the formation of $P$ bodies only in germline or occytes. Taken together, the formation of $\mathrm{P}$ bodies in $C$. elegans appears to be regulated by a specific group of genes.

\section{Functional classes of genes involved in regulating $P$ body formation}

The 216 genes whose inactivations enhance the $P$ body formation in the whole body identified in the screen were categorized into different groups according to their annotated biological functions (Fig. 4G and Table S1). As expected, genes involved in various aspects of RNA metabolism were identified in our screen. RNAi inactivation of a number of genes key to transcription markedly induced the formation of $P$ bodies. These genes include core components of the RNA Pol I, II, and III, transcription initiation and elongation factors (e.g. mdt-15 and spt-5). In addition, we identified genes involved in chromatin remodeling, including histone deacetylase hda-1 and the C. elegans Rb homolog lin-35. RNAi of genes encoding mRNA binding and processing factors also caused an increase in $\mathrm{P}$ body number. These include components involved in splicing, such as lariat debranching enzyme $d b r-1$ and EJC components (Y14/rnp-4 and UAP56/ hel-1). We also identified a group of genes involved in mRNA decay, including the mRNA decapping activator RCK/cgh-1, the CCR4/NOT deadenylase complex (ccr-4 and ntl-2) and the exosome complex (e.g. Rrp40/exos-3 and Rrp42/exos-7). Enhanced formation of $P$ bodies was also observed through loss of function of genes previously shown to be involved in the NMD pathway, including the nuclear cap binding proteins (CBP20/ncbp-2 and CBP80/ncbp-1) and NMD3/T25G3.3, or 

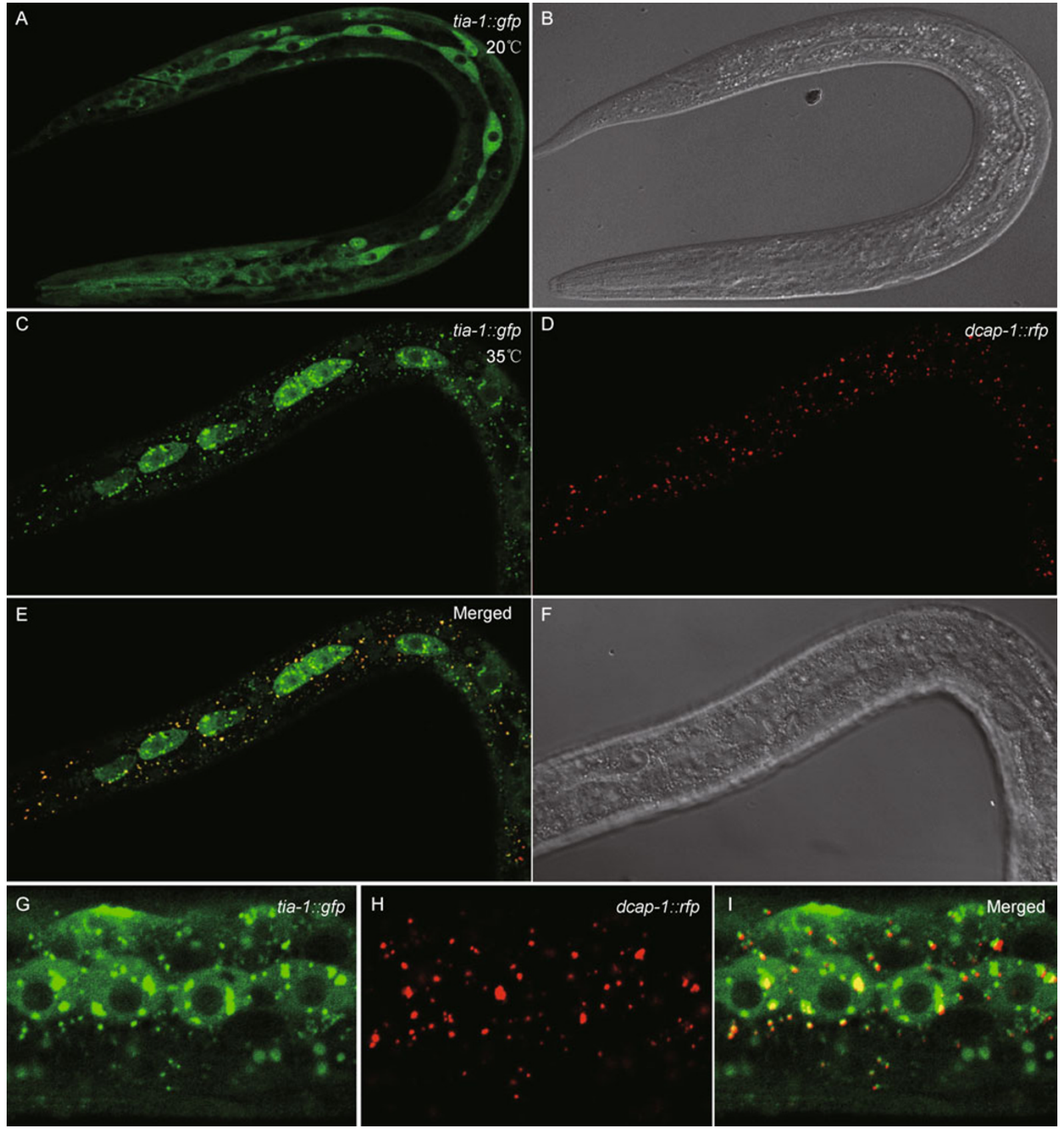

Figure 3. TIA-1 forms granules under stress conditions and is colocalized with $P$ bodies. $(A$ and $B)$ Diffuse TIA-1::GFP signals are present in the cytoplasm, especially in seam cells and hypodermal cells, in a wild type larva at $20^{\circ} \mathrm{C}$. (B) Nomarski image of the animal shown in (A). (C-F) Distribution of TIA-1::GFP (C) and DCAP-1::RFP (D) in a heat shock-treated animal. TIA-1::GFP forms aggregates after the animal was shifted to $35^{\circ} \mathrm{C}$ for one hour $(\mathrm{C})$. The merged images $(\mathrm{E})$ showing the colocalization of TIA-1 bodies and $\mathrm{P}$ bodies. C-F are confocal images. (G-I) Enlarged images showing the colocalization of TIA-1 granules and P bodies. Granules containing only TIA-1::GFP or DCAP-1::RFP are also present. G-I are confocal images.

by inactivation of the $C$. elegans miRNA pathway component, alg-1. Identification of $c g h-1, c c r-4$ and $a l g-1$ in our screen further suggests the specificity of the genes identified in regulating $P$ body formation.

The formation of $\mathrm{P}$ bodies was also regulated by genes involved in protein synthesis and turnover. The largest group of genes identified contains a diverse set of genes involved in protein synthesis, including tRNA synthetases, small and large ribosomal proteins and various translation factors involved in initiation (e.g. subunits of elF-1) and elongation 


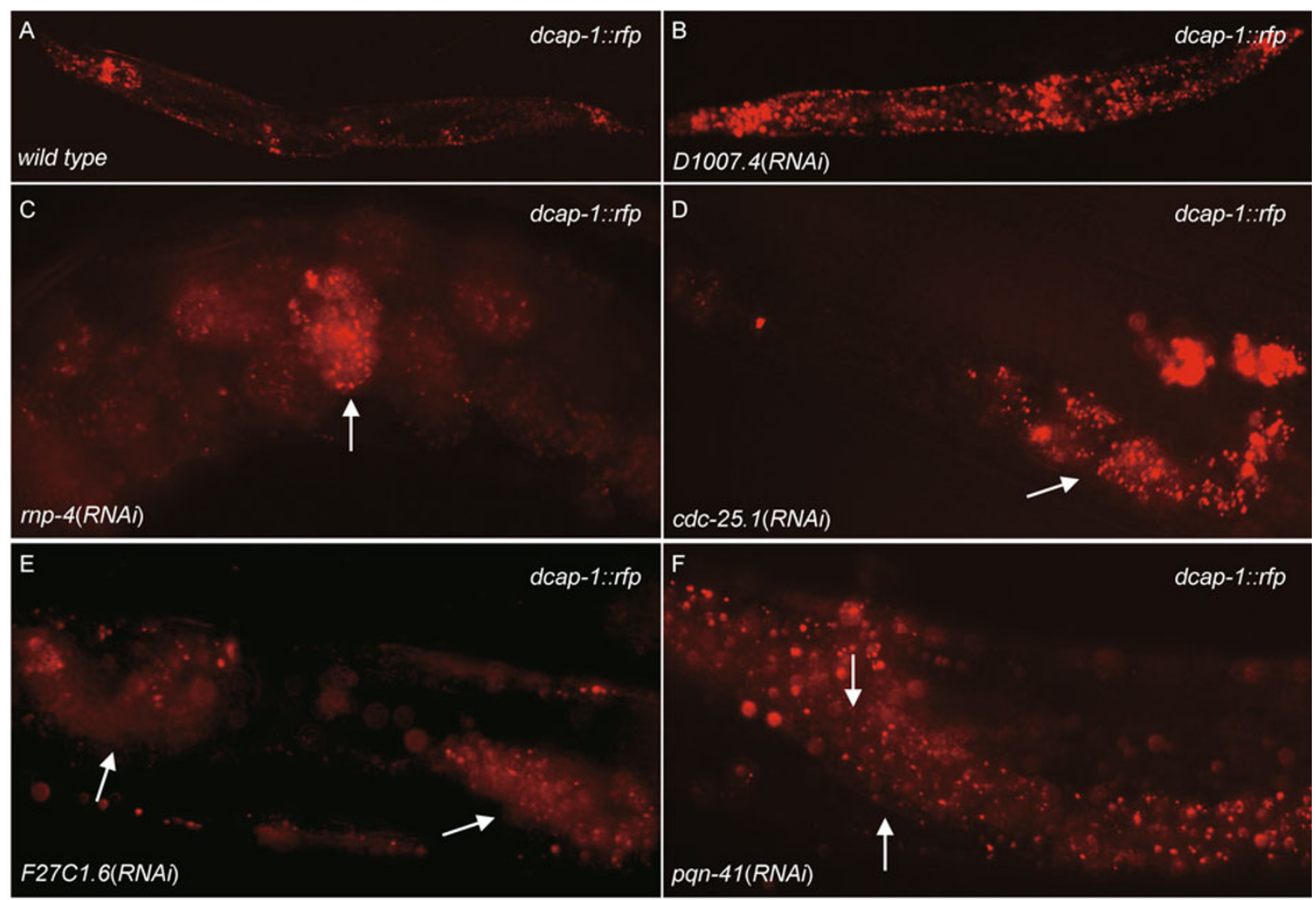

G

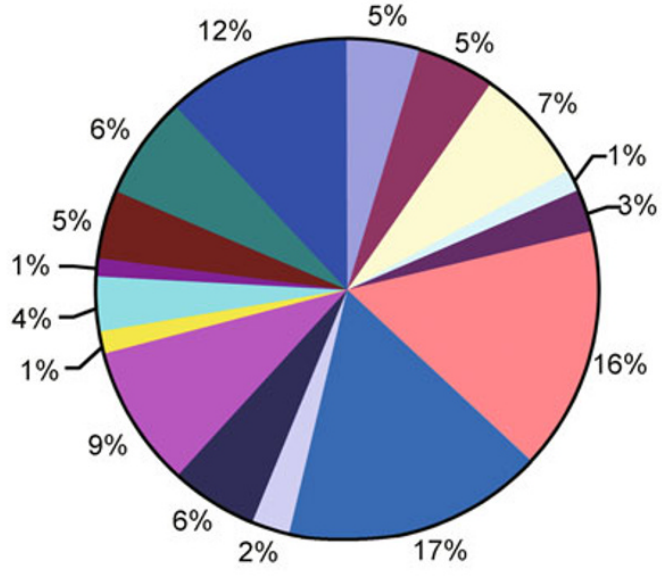

216 genes
口 RNA polymerase subunit (10)

- Transcription factors (11)

口 RNA binding and processing (16)

口 DNA synthesis and repair (3)

- Translation factors (6)

- Ribosomal subunit and tRNA synthases (34)

- Ribosomal biogenesis (36)

口 Protein degradation (5)

- Nuclear transport (12)

口 Protein trafficking (20)

口 Protein modification (3)

口 Mitochondrial proteins (8)

- Cell cycle regulation (2)

- Cytoskeleton and EMC (10)

口 Enzymes (14)

- Others and function unknown (26)

Figure 4. Enhanced formation of DCAP-1::RFP-labeled $P$ bodies by RNAi inactivation of selected genes and their functional classification. (A) Distribution of $\mathrm{P}$ bodies in animals carrying the $d c a p-1:: \mathrm{fp}$ transgene. (B) Enhanced formation of $\mathrm{P}$ bodies in most somatic tissues in D1007.4(RNAi) animals. (C-F) Examples of enhanced formation of $\mathrm{P}$ bodies in specific tissues: embryos in $r n p-4(R N A i)$ animals (arrow in C); germline cells in cdc-25.1(RNAi) and F27C1.6(RNAi) animals (arrow in D and E); intestine in pqn-41(RNAi) animals (arrow in F). (G) Classification of genes regulating the formation of $\mathrm{P}$ bodies in the whole animal according to their general molecular function based on Gene Ontology annotation. The total number of identified genes in each functional class is shown in parentheses. 
(EF-2). Another set of genes encodes factors that control several aspects of ribosomal biogenesis, including rRNA transcription (RNA Pol I and III subunits), modification and processing of pre-rRNAs (e.g. rRNA 2'-O-methyltransferase Fibillarin/fib-1 and H/ACA snoRNP complex components) and intranuclear and nucleo-cytoplasmic transport (e.g. pro-2, SDA-1/pro-3 and eif-6). Components involved in the ubiquitinproteasome degradation system were also recovered in our screen. These include components of the $20 \mathrm{~S}$ core proteasome complex (e.g. pas-6 and pbs-2) and the Cdc48/Ufd1/ $\mathrm{Np} 4$ complex involved in ERAD (ER-associated degradation) (Halawani and Latterich, 2006).

We identified factors that are involved in intracellular trafficking system. These include components functioning in nuclear import and export including nucleoporins (e.g. $n p p-6$ and npp-10), importin (ima-3 and imb-3) factors, exportin (imb-4), nuclear transport factor NTF2/ran-4 and RNA export factor $n x t-1$. Genes involved in protein sorting and intracellular vesicular trafficking were strongly represented in our screen, including those encoding components of the COPI complex (e.g. C13B9.3), clathrin and adaptor protein complex AP-1 (apb-1 and apm-1), SNAREs (syn-3 and snap-1) and vesicle trafficking protein SLY1/F43D9.3. We also identified vacuolar $\mathrm{H}+$ ATPase $\mathrm{V} 0$ and $\mathrm{V} 1$ sector subunits (e.g. vha-17 and -19) and vacuolar assembly/sorting proteins.

Our screen also revealed that inactivation of genes involved in mitochondrial function led to a dramatic increase in $\mathrm{P}$ body number. These include the electron-transport chain subunit (cyc-1 (complex III) and C01F1.2 (complex IV)), subunits of F1F0-ATP synthase (e.g. atp-3), mitochondrial ribosomal protein S18, and TCA-cycle enzymes (e.g. isocitrate dehydrogenase/F43G9.1). This suggests that energy consumption or supply may regulate the assembly of $\mathrm{P}$ bodies.

Other classes of factors that emerged from our screen include cytoskeleton and extracellular matrix components, such as the cell adhesion complex protein byn-1, actin, collagens and intermediate filament proteins. Genes involved in regulating cell cycle progression (e.g. sas-4 and cdc-25), SUMO modification (smo-1 and ubc-9), protein kinases (e.g. kin-2) and phosphatases (e.g. let-92) were also recovered in our screen. We also identified factors involved in various aspects of metabolism, such as carbohydrate metabolism (e.g. C05C8.7), purine metabolism (e.g. W06H3.3) and lipid metabolism (e.g. HMG-CoA synthase/F25B4.6). Twenty-six of the genes uncovered by the screen have no annotated molecular function.

Of the 224 identified genes, 156 have clear human homologs according to gene ontology, suggesting that they may have evolutionarily conserved roles in regulating the formation of $P$ bodies. Taken together, our results suggest that the formation of $\mathrm{P}$ bodies is regulated by diverse cellular processes.

\section{Functional interactions among genes that regulate $\mathbf{P}$ body formation}

The above classification revealed that the formation of $P$ bodies is regulated by genes functioning in various biological processes. To obtain a global view of functional interactions among genes that regulate $\mathrm{P}$ body formation, we identified possible functional interactions among these genes. The genome-wide functional interaction map in C. elegans was predicted according to the computational integrated interaction data, gene expression data, phenotype and functional annotation data (Zhong and Sternberg, 2006). As a control, we randomly selected three groups of 216 genes from the same gene library used in this study and detected two interactions (edges) among four genes (nodes) in two of three groups and no interaction in another group. However, among the 216 genes whose inactivations enhanced the formation of $P$ bodies, we found that 133 genes (nodes) formed at least one interaction (edge) with other genes. A total of 617 edges were identified $(p<0.05)$ (Fig. S1). Thus, the formation of $\mathrm{P}$ bodies is regulated by genes that are functionally related or involved in the same process.

\section{Regulation of NMD by a subset of identified genes that modulates $\mathrm{P}$ body formation}

Loss of function of NMD factors, including UPF2 and UPF3, leads to enhanced formation of $P$ bodies in yeast (Sheth and Parker, 2006). Thus, mutants with increased $P$ body number could be involved in regulation of NMD in C. elegans. We tested this by examining the effect of the identified RNAi clones on the expression of gfp::bro-1(PTC), whose expression is regulated by NMD. Wild type gfp::bro-1 was expressed in seam cells at larval and adult stages (Fig. 5A) (Xia et al., 2007), while gfp::bro-1(PTC), containing a premature stop codon in the last exon of bro-1, was not detectable in larvae (Fig. 5B). Consistently, the level of gfp::bro-1(PTC) mRNA was dramatically decreased compared to that of gfp::bro-1 (Fig. 5E). Knockdown of key regulators of NMD, including $s m g-1,-4$, or -5 , restored the expression of gfp::bro-1(PTC) in larvae and concomitantly led to an increase in its mRNA level (Fig. 5C and 5E). Thus, gfp::bro-1(PTC) mRNA is a target of NMD. Of the 216 genes identified above, we found that gfp:: bro-1(PTC) showed obvious expression in late larvae in 83 RNAi clones (the ratio of animals expressing the reporter $\geqslant$ 0.25) (Fig. 5D, Table 1 and Table S1). Semi-quantitative RTPCR analysis showed an increase in gfp::bro-1(PTC) mRNA level in the tested mutants (Fig. 5E). Of the 83 identified genes, knockdown of 68 genes by RNAi feeding caused larval arrest or sterility. None of the 83 RNAi clones increased the expression of wild type gfp::bro-1 reporter, suggesting that restoration of gfp::bro-1(PTC) in seam cells is not caused by upregulation of bro-1 transcripts. 

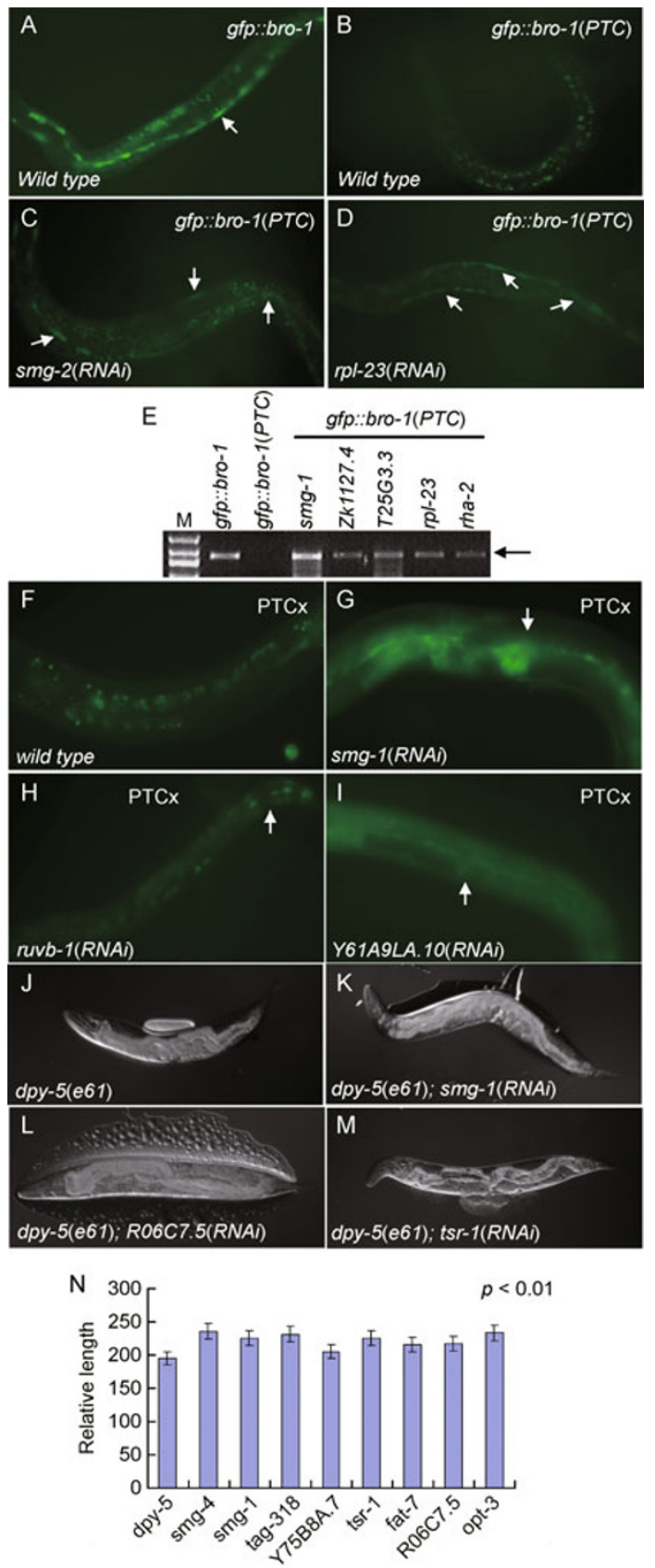

Figure 5. Identification of genes with a role in regulation of NMD. (A) Expression of the gfp::bro-1 reporter in seam cells (arrow). (B) Expression of the gfp::bro-1(PTC) reporter is not detectable in late larvae. gfp::bro-1(PTC) contains a premature termination codon at the 91st codon in BRO-1, which is 152 amino acids long. The irregular particles are gut autofluorescence. (C) gfp::bro-1(PTC) is expressed in seam cells in smg-2(RNAi) mutant larvae (arrows). (D) gfp::bro-1(PTC) is expressed in seam cells in rpl-23(RNAi) mutant larvae (arrows). (E) The mRNA level of gfp::bro-1(PTC) is increased after RNAi inactivation of $s m g-1$ and other genes identified in this study. Arrow indicates the gfp::bro-1 mRNA. (F) PTCx is weakly expressed in a wild type animal. (G) RNAi inactivation of smg-1 dramatically increases the expression level of PTCx. (H and I) RNAi inactivation of ruvb-1 $(\mathrm{H})$ and Y61A9LA.10 (I) lead to the increased expression of PTCx. ( $\mathrm{J}$ and K) The Dpy phenotype caused by the dpy-5(e61) mutation (J) is suppressed by smg-1(RNAi) (K). ( $\mathrm{L}$ and M) Suppression of the Dpy phenotype of $d p y-5(e 61)$ by RNAi inactivation of $R 06 C 7.5(\mathrm{~L})$ and tsr-1 (M). (N) The relative body size of $d p y-5(e 61)$ mutants treated with various RNAi clones. The average size of adult animals is derived from at least eight animals for each RNAi clone. 


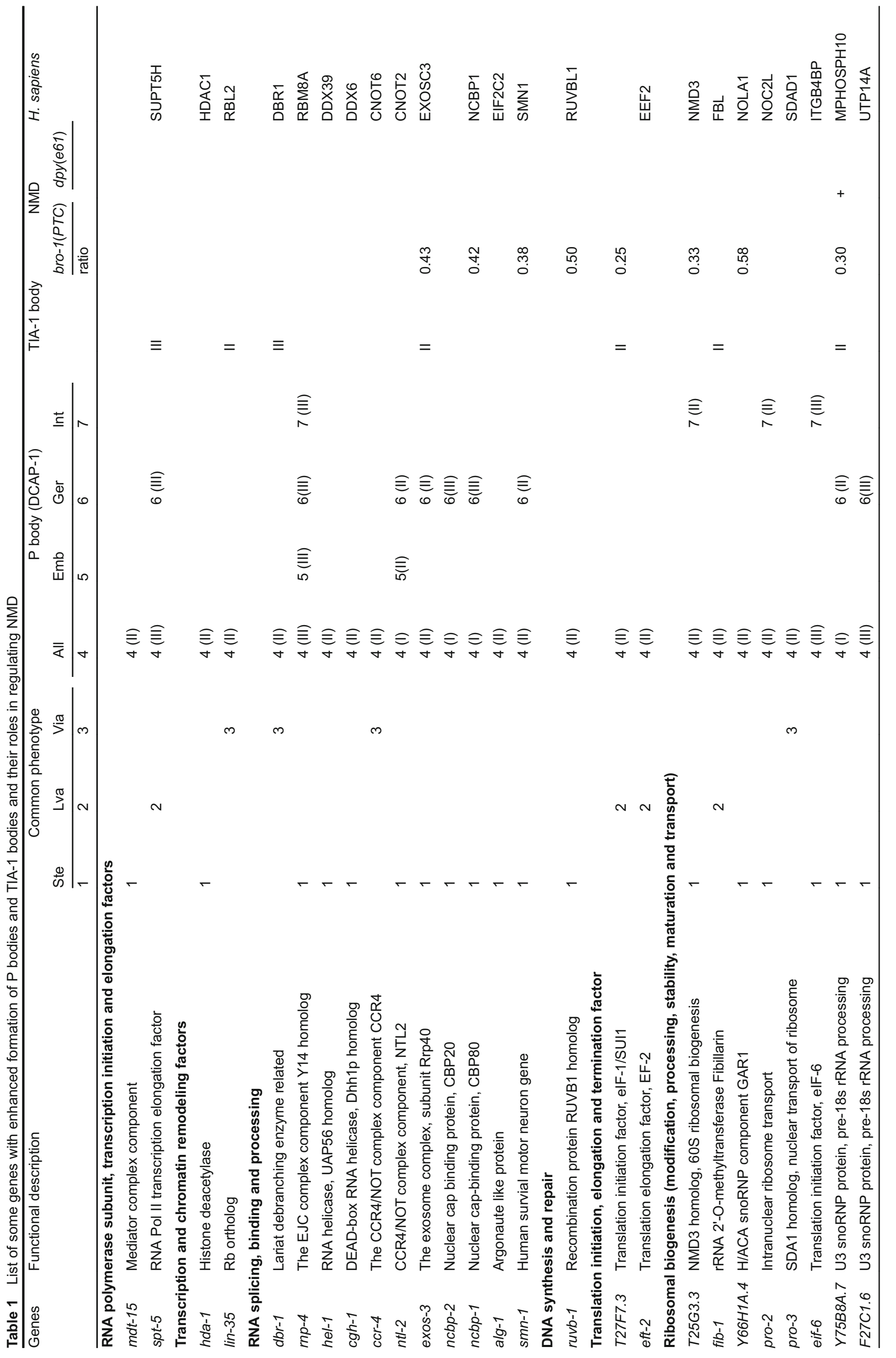




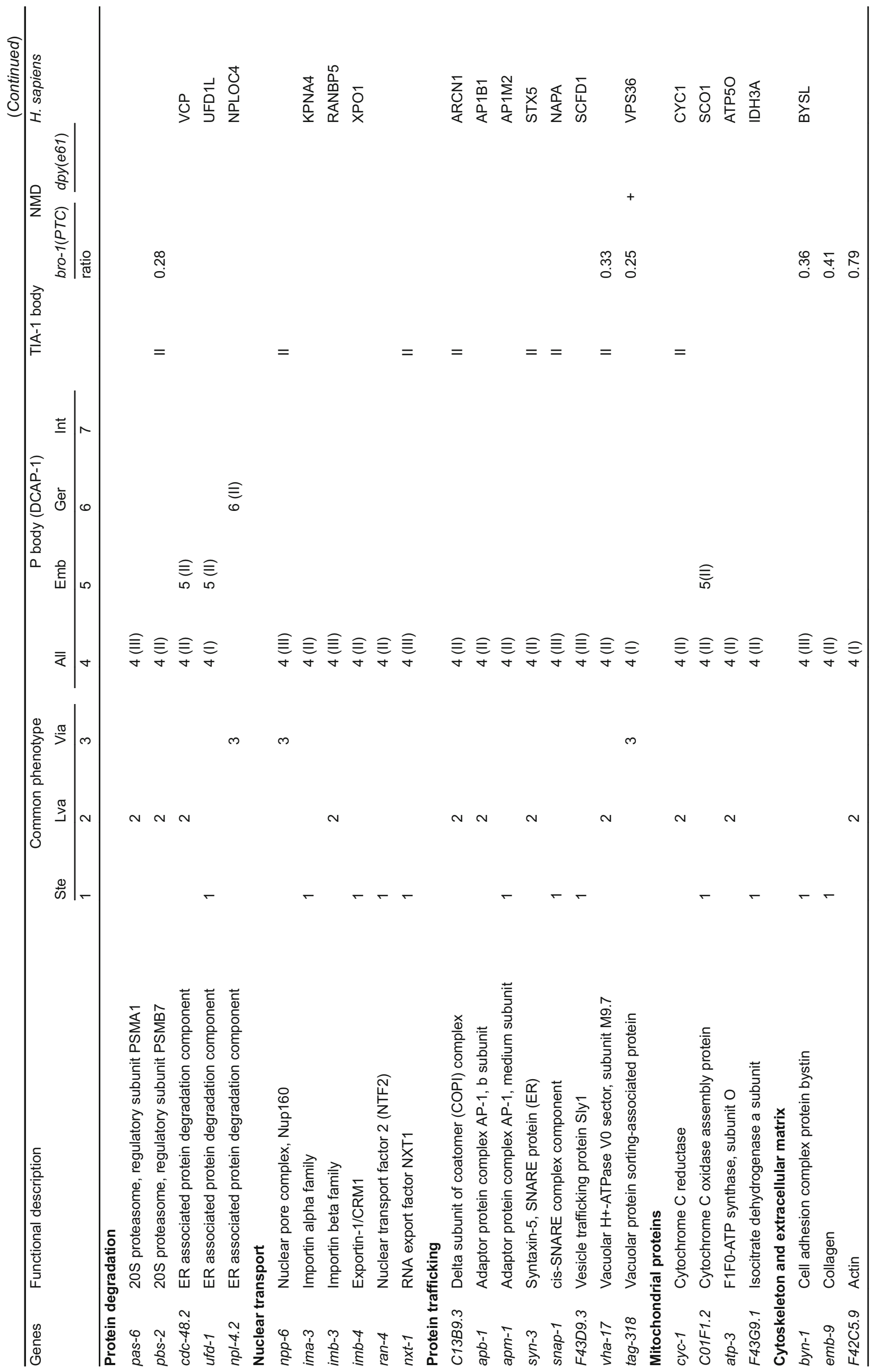




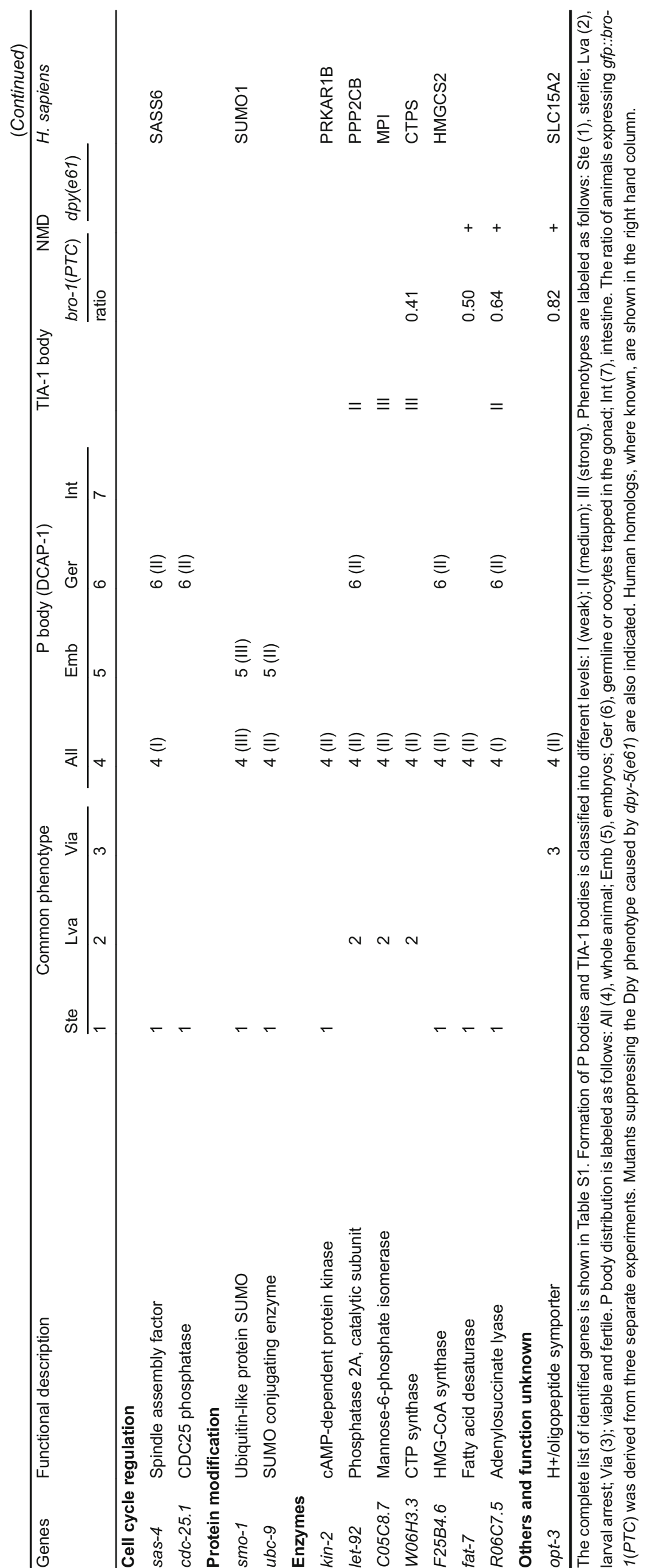


We further examined the effect of the identified $83 \mathrm{RNAi}$ clones on the expression of a previously characterized NMD target, PTCx (Longman et al., 2007). The PTCx reporter contains a GFP::LacZ fusion gene that is driven by a ubiquitous expressed promoter and a PTC is placed in the first exon of lacZ (lacZ containing four synthetic introns) (Longman et al., 2007). Thus, gfp is only weakly expressed in animals carrying the PTCx transgene (Fig. 5F). Mutations in the NMD pathway, such as smg-2, lead to the stabilization of PTCx mRNA and restore the expression of GFP::LacZ (Fig. 5G). We found that all 83 RNAi clones identified above elevated the expression level of PTCx, in a magnitude similar to smgl-1 (Fig. 5H and 5I) (Longman et al., 2007). Thus, the identified 83 RNAi clones appear to suppress the degradation of PTC containing mRNAs in a gene-nonspecific manner.

We next determined whether the RNAi clones with increased expression level of gfp::bro-1(PTC) and PTCx also suppressed the Dpy phenotype associated with $d p y-5$ (e61), a well-characterized target of NMD (Hodgkin et al., 1989). Of the 83 genes, only 61 RNAi inactivations that gave rise to late larvae or adult animals could be scored for suppression of the Dpy phenotype. RNAi inactivation of six genes, tag-318, Y75B8A.7, tsr-1, fat-7, R06C7.5 and opt-3, significantly increased the body size of $d p y-5(e 61)$ animals (Fig. 5J and 5N, Table 1 and Table S1). However, RNAi inactivation of these six genes did not suppress the Dpy phenotype caused by the null $d p y-5(e 907)$ mutation. Thus, the mechanism these RNAi clones suppress $d p y-5(e 61)$ is most likely by stabilization of $d p y-5(e 61)$ mRNA, but not in a $d p y-5$-independent manner. Compared to the expression of gfp::bro-1(PTC) and PTCx reporters, the small number of gene inactivations suppressing $d p y-5(e 61)$ could be partly due to difficulties in discerning subtle changes in body size. Furthermore, the number of introns and the location of the PTC relative to the $3^{\prime}$ end of mRNA, which have been shown to have an effect on NMD efficiency, are different in gfp::bro-1 (PTC) and dpy-5(e61). gfp::bro-1(PTC) contains five introns and the PTC is located in the last exon, while $d p y-5$ contains no introns and the PTC is located 81 amino acids upstream of normal stop codon.

Among the 83 genes that regulate the expression of $g f p::$ bro-1(PTC), we identified C. elegans genes whose yeast or mammalian homologs have been implicated in NMD, including elF-1/T27F7.3, nuclear cap binding protein CBP80/ncbp1, the exosome complex and NMD3/T25G3.3 ( $\mathrm{He}$ and Jacobson, 1995; Cui et al., 1999; Hosoda et al., 2005; Houseley et al., 2006). Consistent with previous studies, inactivation of components of EJC did not affect the expression of gfp::bro-1(PTC). However, smn-1, encoding the human motor neuron gene that has been shown to be involved in RNA processing, affected the stability of gfp::bro-1 (PTC) mRNA. 50 out of 83 identified genes are involved in protein synthesis, including rRNA production (subunits of Pol I and Pol III), translation initiation factors, tRNA synthetases, ribosomal proteins and ribosomal biogenesis factors (Fig. 6A). This suggests that reduced translation efficiency impairs mRNA surveillance. Mutations in components of the proteasome degradation system, including $p b s-2$, also led to defects in NMD. Vacuolar-mediated trafficking factors vha-17 and tag-318 were also recovered in our screen. Cytoskeleton and extracellular matrix components, including byn-1, emb-9, and actin/F42C5.9, also regulated the stabilization of gfp::bro1(PTC) mRNA. Identification of CTP synthase/W06H3.3, adenylosuccinate lyase/R06C7.5 and fatty acid desaturase fat-7 indicates that NMD is coupled with nucleotide and lipid metabolism. Therefore, the efficiency of NMD is regulated by many cellular functions including translation, protein turnover and cytoskeleton dynamics.

Next, we examined possible interactions among the 83 genes that regulate the expression of gfp::bro-1(PTC) and PTCx. 53 have functional interactions and 199 interactions (edges) were found in total (Fig. 6B). As a control, we randomly selected three groups of 83 genes from the same gene library, one interaction (edge) was observed between two genes (nodes) in one group, and no interaction was found in other two groups. The functional interaction map revealed that T25G3.3 directly interacts with 14 genes, which further interact with another 28 genes. T25G3.3 encodes the $C$. elegans homolog of NMD3, which has been shown to function in NMD by interacting with UPF1 (He and Jacobson, 1995). NMD3 is also essential for nuclear export of the 60S ribosomal subunit (Hedges et al., 2006). As expected, T25G3.3 interacts with ribosomal subunits (e.g. RPA-0, RPL-37, and RPS-2), factors involved in ribosomal biogenesis and nuclear export (H06I04.3, Y66H1A.4, Y61A9LA.10, $\mathrm{K} 12 \mathrm{H} 4.3$ and NST-1). The interaction map also revealed some novel interactions, such as the interaction between T25G3.3 with RUVB-1, a protein involved in DNA synthesis and repair, and with the cell adhesion protein BYN-1. Taken together, this interaction network provides a global view of genes that may work together in controlling NMD.

\section{Regulation of RNAi by a subset of identified genes that regulates $P$ body formation}

We next determined whether the 216 genes whose inactivations lead to an increase in $\mathrm{P}$ body number also play a role in RNAi. Previous studies have shown a complete functional overlap of factors required for RNAi and silencing of the expression of scm::gfp transgene (Kim et al., 2005). scm::gfp is expressed in hypodermal seam cells. However, its expression is almost completely silenced in RNAi-sensitive mutants, including eri-1 and rrf-3 (Fig. 7A and 7D), while inactivation of genes required for RNAi restores the expression of scm::gfp in eri-1 and rrf-3 mutants (Fig. 7B) (Simmer et al., 2002; Kennedy et al., 2004). Of the 216 genes, we found that RNAi of 12 genes restored the expression of scm::gfp in 
A

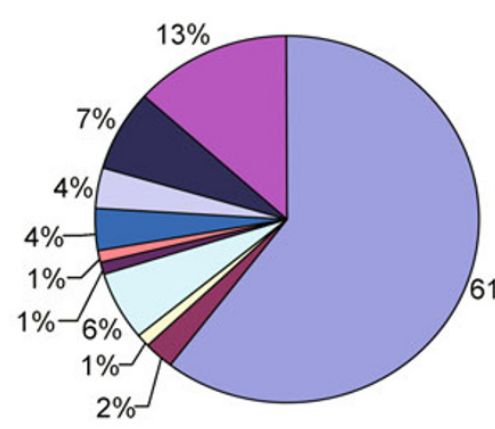

$\square$ Protein synthesis $(50)$

- Transcription factors (2)

$\square$ DNA synthesis and repair (1)

$\square$ RNA binding and processing (5)

- Protein degradation (1)

$\square$ Nuclear transport (1)

a Protein trafficking (3)

$\square$ Cytoskeleton and EMC (3)

- Enzymes (6)

- Others and function unknown (11)

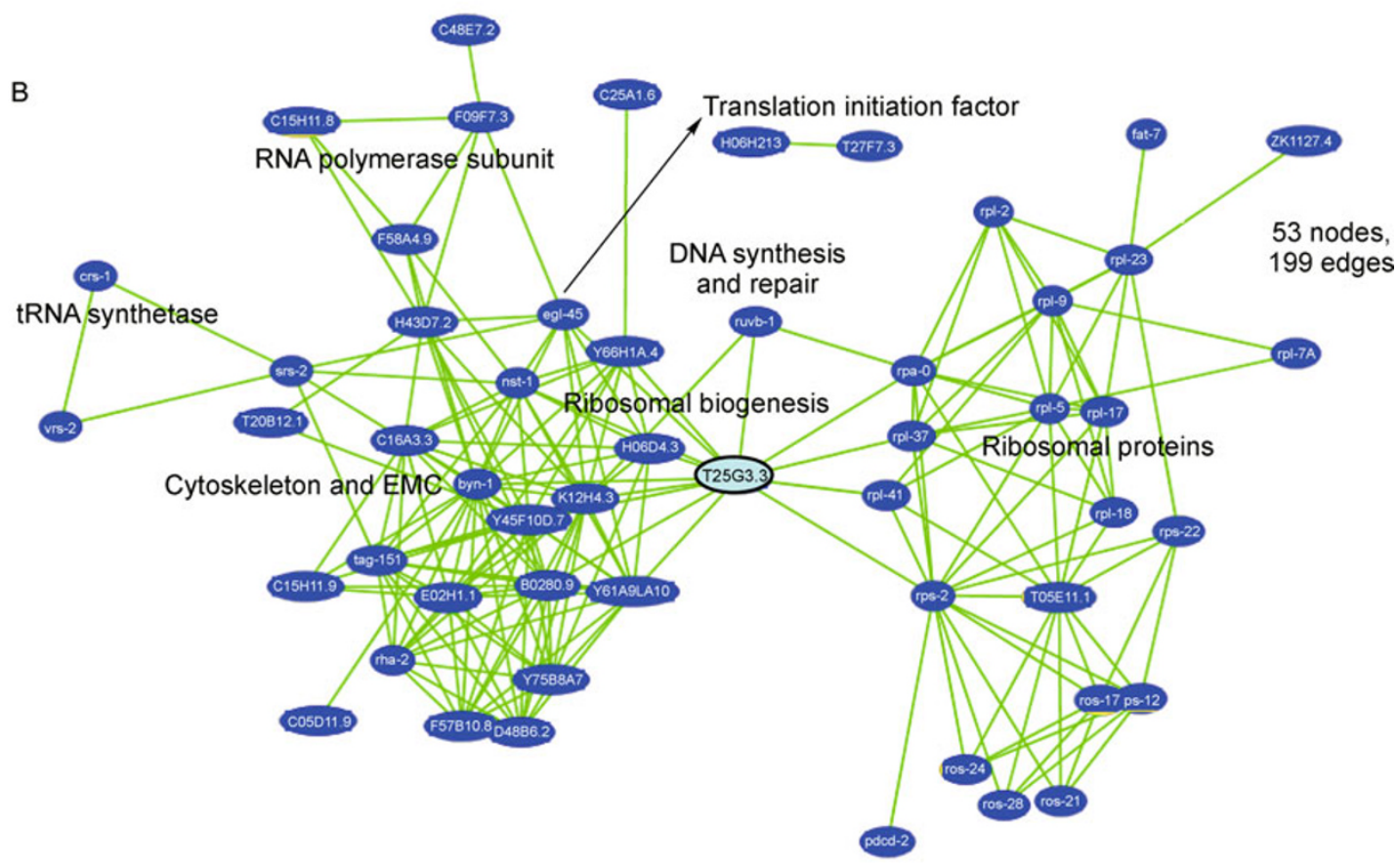

Figure 6. Classification of genes involved in NMD and their functional interactions. (A) Functional classification of genes with a role in NMD. The total number of identified genes in each functional class is shown in parentheses. (B) Functional interactions among genes whose inactivations caused defects in NMD. Of the 83 identified genes, 53 genes (nodes) interact with at least one other gene. 199 edges represent the total number of interactions among the 53 genes. T25G3.3 is highlighted in red. The biological function of neighboring nodes is indicated.

rrf-3 mutants (Fig. 7C and 7G). Among the 12 genes identified, 8 were further confirmed to be involved in RNAi pathway using the RNAi sensor strain, in which the scm::gfp reporter is silenced by a transgene that expresses gfp dsRNA hairpin (Kim et al., 2005). Of the 12 genes that affect the silencing of scm::gfp, 7 are involved in protein synthesis, including the ribosomal protein C37A2.7/rpl-P2 and six ribosomal biogenesis factors $(H 06104.3, I p d-6$, eif-6, F11A10.7, Y45F10D.7 and C15H11.9), which are predicted to form an interaction network (Fig. $7 \mathrm{H}$ ). Of these ribosomal biogenesis factors, eif- 6 has been demonstrated to be required for miRNA-mediated RNA degradation (Basu et al.,
2001; Chendrimada et al., 2007). Therefore, RNAi may be impaired by defects in the formation of functional ribosomal subunits. Other identified genes include nuclear transport components (npp-4 and ima-3) and CTP synthase/W06H3.3.

Amongst the 216 genes regulating $P$ body formation, we also identified 9 mutants in which the expression of scm::gfp was greatly reduced (Fig. 7E, 7F and 7I). Simultaneously depleting the activity of mut-2 or mut-7, both of which are essential components of the RNAi pathway (Ketting et al., 1999; Chen et al., 2005), restored the expression of scm::gfp in those mutants, suggesting that the reduced expression level of $s c m:: g f p$ was likely due to enhanced RNAi sensitivity 

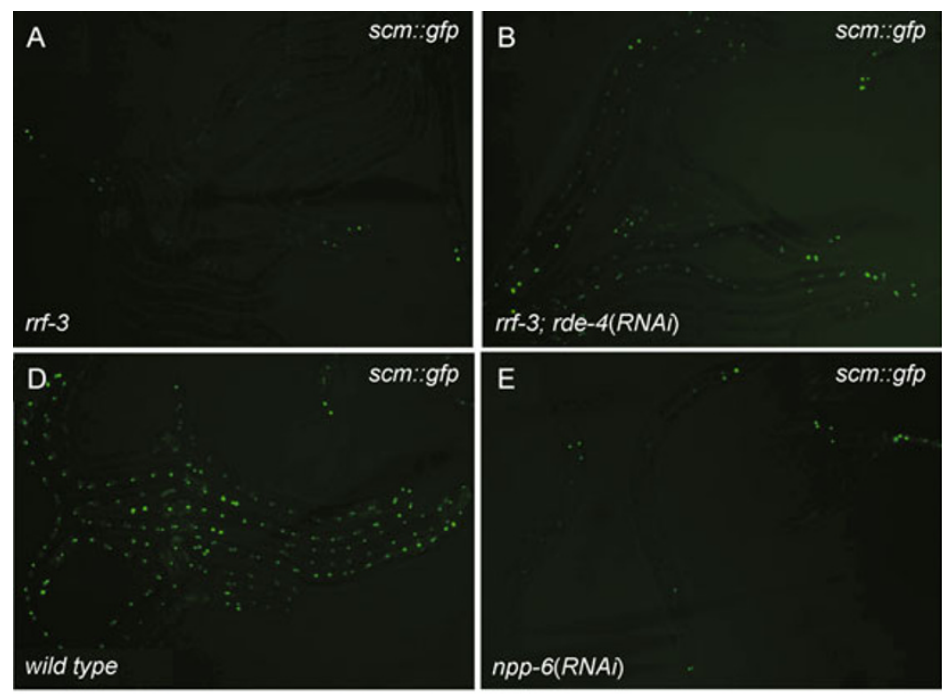
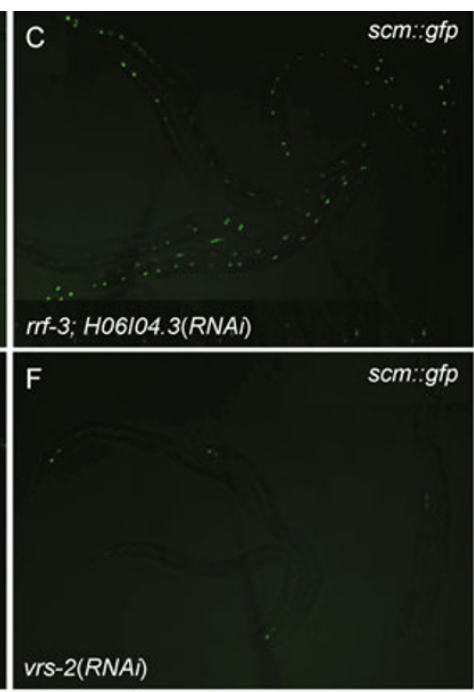

G

\begin{tabular}{|c|c|c|c|c|}
\hline \multirow[t]{2}{*}{ Genes } & \multirow{2}{*}{ Functional description } & \multicolumn{3}{|c|}{ NMD } \\
\hline & & scm::gfp; rrf-3 & $\frac{\text { gfp: bro-1(PTC) }}{\text { (ratio) }}$ & RNAi sensor strain \\
\hline$\overline{F 11 A 10.7}$ & pre-rRNA processing & +++ & & + \\
\hline Y $45 F 10 D .7$ & U3 snoRNP protein & + & 0.57 & + \\
\hline$n p p-4$ & Nuclear pore complex, Nup98 & ++ & & + \\
\hline$i m b-4$ & Exportin-1/CRM1 & + & & \\
\hline ima-3 & Importin alpha family & +++ & & + \\
\hline eif-6 & Translation initiation factor, elF-6 & ++ & & + \\
\hline C37A2.7 & Ribosomal protein, large subunit P2 & +++ & & + \\
\hline C15H11.9 & Nuclear export of $60 S$ pre-ribosome subunit & +++ & 0.84 & + \\
\hline ipd-6 & $60 \mathrm{~S}$ ribosomal biogenesis & +++ & & \\
\hline Ho6/04.3 & SAM-dependent rRNA methyltransferase & +++ & 0.37 & + \\
\hline$a b c x-1$ & $A B C$ transporter & ++ & & \\
\hline W06H3.3 & CTP synthase & ++ & 0.41 & \\
\hline
\end{tabular}

I

\begin{tabular}{|c|c|c|c|c|}
\hline Genes & Functional description & $s c m:: g f p$ & $s c m:: g f p ; m u t-2$ & $s c m:: g f p ; m u t-7$ \\
\hline$\overline{n p p-6}$ & Nuclear pore complex, Nup160 & --- & $\mathrm{N}$ & $\mathrm{N}$ \\
\hline Y61A9LA.9 & Unknown function & -- & $\mathrm{N}$ & $\mathrm{N}$ \\
\hline eif-3.F & Translation initiation factor, elF-3F & - & $\mathrm{N}$ & $\mathrm{N}$ \\
\hline ars-2 & Alanyl tRNA synthetase & -- & $\mathrm{N}$ & $\mathrm{N}$ \\
\hline vrs-2 & Valyl tRNA synthetase & - - - & $\mathrm{N}$ & -- \\
\hline grs-1 & Glycyl tRNA synthetase & - - & $\mathrm{N}$ & - - \\
\hline F43D9.3 & Vesicle trafficking protein Sly1 & - & $\mathrm{N}$ & $\mathrm{N}$ \\
\hline syn-3 & Syntaxin-5, SNARE protein (ER) & - - & $\mathrm{N}$ & $\mathrm{N}$ \\
\hline$n s f-1$ & AAA+-type ATPase, vesicle fusion ATPase & $-\ldots$ & $\mathrm{N}$ & $\mathrm{N}$ \\
\hline
\end{tabular}

$\mathrm{H}$

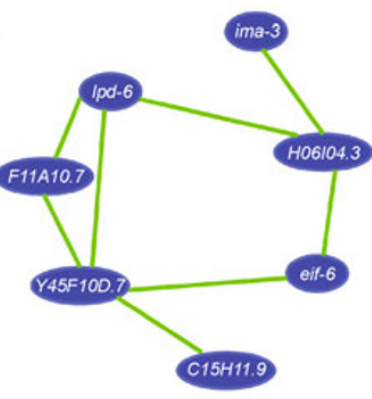

J

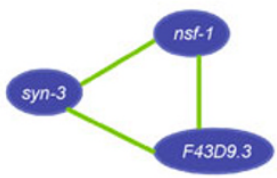

Figure 7. Identification of genes with a role in regulation of RNAi. (A) Expression of scm::gfp in seam cells is almost completely silenced in RNAi-sensitive rrf-3 mutant animals. (B) Expression of scm::gfp in rrf-3 mutants is restored by simultaneously depleting the activity of $r d e-4$, an essential component of the RNAi pathway. (C) Expression of scm::gfp in rrf-3 mutants is restored by RNAi inactivation of H06I04.3. (D) Strong expression of scm::gfp in seam cells in a wild type animal. (E and F) Expression of scm:: gfp is significantly reduced in $n p p-6(R N A i)(\mathrm{E})$ and $v r s-2(R N A i)(\mathrm{F})$ animals. $(\mathrm{G})$ List of 12 genes whose inactivations restore the expression of scm::gfp in rrf-3 mutants. The increased expression level of the reporter in RNAi inactivation of the genes is classified into three levels:,+++ , and +++ , where +++ indicates that the expression level of $s c m:: g f p$ is as strong as that in wild type animals carrying the scm::gfp transgene. Inactivation of 8 of these genes caused increased expression of scm::gfp in the RNAi sensor strain. The ratio of animals expressing gfp::bro-1(PTC) in these genes is also indicated. $(\mathrm{H})$ Of the 12 genes required for RNAi, seven form a functional network. (I) List of genes whose inactivations reduce the expression of $s c m:: g f p$. The reduced expression level of $s c m::$ gfp in the RNAi clones is classified into three levels: -, --, and ---, where --- indicates that the expression of the reporter is almost abolished. N: No change in the expression level of $s c m:: g f p$ compared to that in wild type animals carrying the scm::gfp transgene. The reduced expression of $s c m:: g f p$ in the identified RNAi clones is restored by simultaneously depleting the activity of mut-7 or mut2, except that scm::gfp is still weakly expressed in grs-1; mut-7 and vrs-2; mut-7 mutants, suggesting that the role of grs-1 and vrs-1 in regulating the expression of $s c m:: g f p$ is epistatic to mut-7. (J) syn-3, nsf-1 and F43D9.3 are predicted to interact with each other. 
(Fig. 7l). The identified genes include factors involved in nuclear transport (npp-6), protein synthesis and vesiclemediated trafficking (sly1/F43D9.3, syn-3 and nsf-1). Components involved in translation include tRNA synthetases (grs-1, ars-2 and vrs-2) and translation factor (elF-3F), suggesting that active translation may protect RNA from RNAi-mediated cleavage. The identification of syn-3, F43D9.3 and nsf-1 (Fig. 7J), which are required for vesicular transport between the ER and the Golgi complex, suggests a role for the intracellular vesicular trafficking network in regulating RNAi efficiency.

\section{Regulation of the formation of stress granules by a subset of identified genes that regulate $P$ body formation}

We next examined the role of identified genes in regulating the formation of TIA-1 bodies. Of the 224 genes whose inactivations enhanced the formation of $\mathrm{P}$ bodies in the whole animal or in specific tissues, we found that RNAi of 76 genes enhanced the formation of TIA-1 bodies. Consistent with that DCAP-1 are colocalized with TIA-1 bodies, the majority of the TIA-1 bodies were colocalized with $P$ bodies in the RNAi clones (Fig. 8A-8D). The formation of stress granules in $C$. elegans is regulated by genes with diverse functions, including in ribosome biogenesis, RNA splicing, translation and protein degradation (Fig. 8E).

\section{DISCUSSION}

\section{Regulation of the formation of $P$ bodies in C. elegans, yeast and mammalian cells}

In this study, we revealed that the formation of $\mathrm{P}$ bodies in $C$. elegans is regulated by various biological processes, including transcription, translation, cytoskeleton, nuclear transport, intracellular vesicular trafficking, the ubiquitin proteasome pathway and mitochondrial maintenance. Defects in these processes could directly regulate the formation of $\mathrm{P}$ bodies or through regulating mRNA metabolism. Previous studies have demonstrated an extensive link between mRNA degradation and other biological processes. For example, the CCR4/NOT deadenylase complex represses the activity of RNA Polymerase II (Liu et al., 2001). The RNA polymerase subunit Rbp4 is required for deadenylation and decay of a subset of transcripts that encodes proteins involved in protein synthesis (Lotan et al., 2005). Inhibition of translation elongation has been shown to stabilize mRNA (Parker and Sheth, 2007). Furthermore, mutations in MRT4, GRC5 (L9 ribosomal protein) and THS1 (threonyl-tRNA synthesase) genes result in defects in mRNA turnover in yeast (Zuk et al., 1999). The cytoskeleton structure also has an effect on the stabilization of specific mRNAs. Loss of function of SLA2, encoding an actin-binding talin-like domain containing protein, causes defects in the mRNA turnover (Zuk et al., 1999). Another possibility is that defects in these processes cause cellular stresses, which could regulate the movement of mRNAs into $P$ bodies, allowing the selective expression of proteins required for alleviating the stress, while retaining the majority of the cytoplasm pool of mRNAs for late reuse and recovery from stress. However, it appears that not all identified RNAi clones enhanced the formation of $\mathrm{P}$ bodies through the same mechanism, as only a subset of genes, when inactivated, leads to the formation of SGs. In yeast, stress treatment results in increased number of $\mathrm{P}$ bodies (Teixeira and Parker, 2007). However, in mammalian cells, bulk mRNAs are stored in stress granules upon stress treatment (Kedersha et al., 2005). Therefore, regulation of formation of $P$ bodies in $C$. elegans resembles that in yeast and the formation of SGs in mammalian cells.

Studies in yeast and mammalian cells have demonstrated an essential role of factors regulating the mRNA synthesis and degradation in the formation of $\mathrm{P}$ bodies. Inhibition of $\mathrm{Pol}$ II transcription, repression of translation elongation, inhibition of mRNA degradation at a step prior to decapping, or inhibition of the RNAi and miRNA pathway results in the disappearance of $P$ bodies, while repression of translation initiation or inhibition of mRNA decay at a step at or after decapping induces the formation of $\mathrm{P}$ bodies (Parker and Sheth, 2007). In this study, we revealed a remarkable difference in the regulation of the formation of $\mathrm{P}$ bodies in C. elegans compared to yeast or mammalian cells. Inhibition of RNA Pol II activity, defects in translational elongation, or loss of function of the mRNA decay components RCK and CCR4, or inactivation of the miRNA silencing pathway component Argonaute, all lead to the enhanced formation of $P$ bodies in $C$. elegans. One possible explanation for this could be that RNAi feeding only partially reduces the gene activity. Alternatively, inactivation of these genes elicits a cellular stress, which in turn regulates the $\mathrm{P}$ body formation.

\section{Regulation of NMD by various biological processes}

Our study demonstrated a conserved role of the CBP20/80 nuclear cap binding complex and the $3^{\prime}$ to $5^{\prime}$ exosome complex in NMD in $C$. elegans and in mammalian cells (Hosoda et al., 2005; Houseley et al., 2006). CBP80 augments the efficiency of NMD by interacting with UPF1 and promoting the interaction of UPF1 and UPF2. The NMD pathway has also been shown previously to be regulated by translation, which could be required for the NMD machinery to detect the target mRNA. NMD is blocked by cycloheximide or nonsense suppressor tRNAs. The NMD pathway in yeast is suppressed by mutations in SUI1, which encodes the elF1 homolog that is involved in the reorganization of the AUG codon during translation initiation and maintenance of the appropriate reading frame during translation elongation (Cui et al., 1999). We showed that NMD is impaired by inactivation 

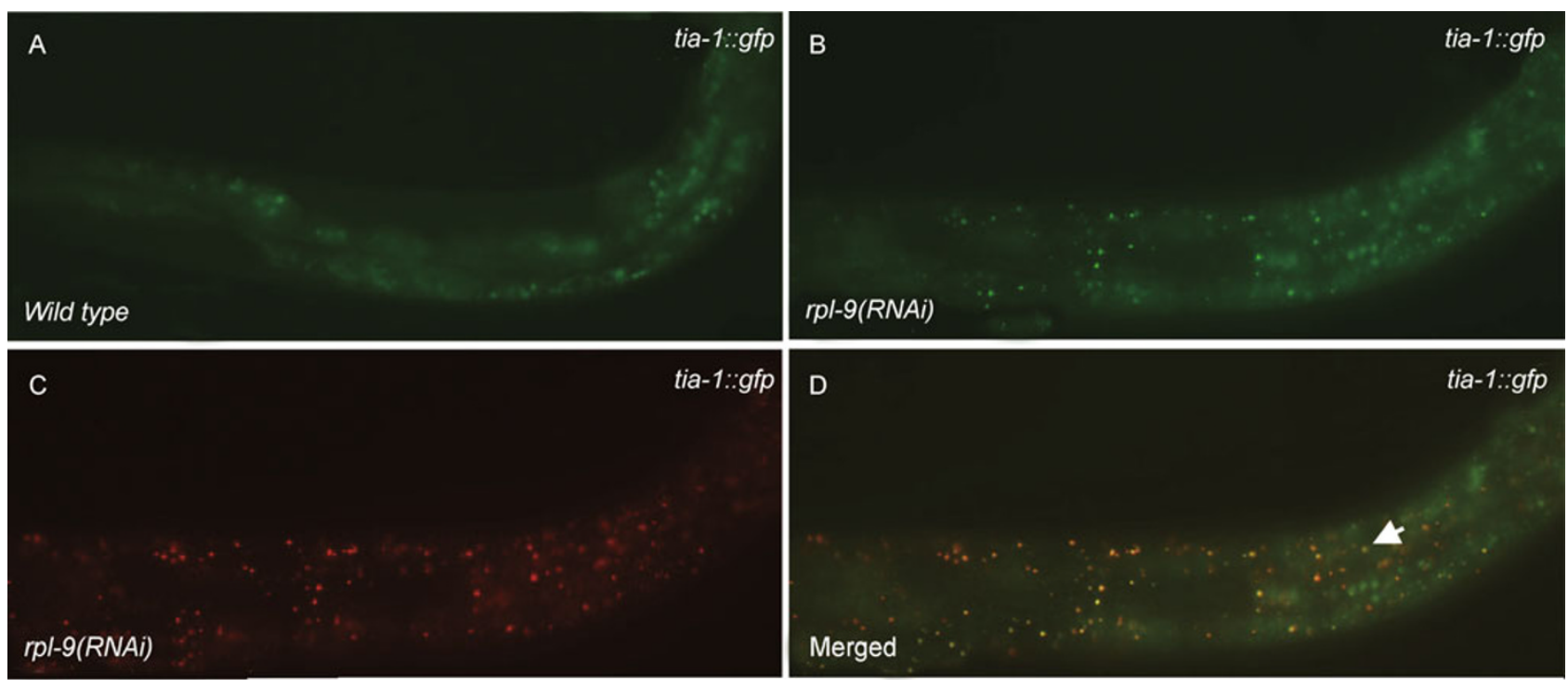

E

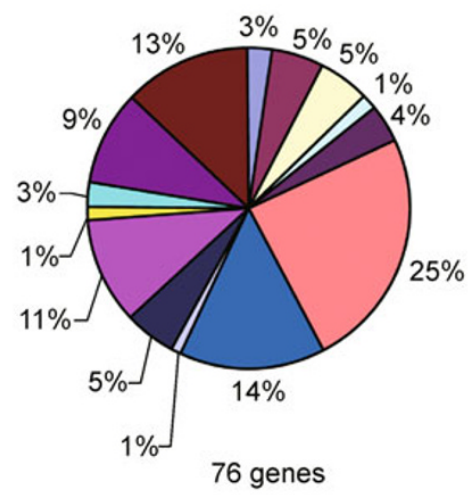

$\square$ RNA polymerase subunit (2)

$\square$ Transcription factors (4)

$\square$ RNA binding and processing (4)

$\square$ DNA synthesis and repair (1)

$\square$ Translation initiation factors (3)

$\square$ Ribosomal proteins and tRNA synthases (18)

$\square$ Ribosomal biogenesis (11)

$\square$ Protein degradation (1)

$\square$ Nuclear transport (4)

$\square$ Protein trafficking (8)

$\square$ Mitochondrial proteins (1)

$\square$ Cytoskeleton and ECM (2)

Enzymes (7)

Others and function unknown (10)

Figure 8. Regulation of the formation of TIA-1 bodies. (A) tia-1::gfp is weakly expressed and homogenously distributed in the cytoplasm in a wild type animal. The irregular particles are gut autofluorescence. (B and E) Formation of TIA-1::GFP bodies (B) and P bodies $(C)$ in $r p l-9(R N A i)$ animals. The merged image (D) shows that the majority of TIA-1 bodies are colocalized with $P$ bodies in RNAi clones (arrow). (E) Functional classification of the genes regulating the formation of TIA-1 bodies. The total number of identified genes in each functional class is shown in parentheses.

of components in multiple steps of protein synthesis, including rRNA production, translation initiation factors, tRNA synthetases, ribosomal proteins and ribosomal biogenesis factors, revealing an extensive link between translation and NMD. One of the factors identified is T25G3.3, encoding the C. elegans NMD3 homolog. NMD3 directly interacts with UPF1 and mutations in NMD3 cause synthetic lethal with an Xrn1 mutant (He and Jacobson, 1995; Johnson, 1997). Mutant alleles of NMD3 also show altered rRNA stability. NMD3 colocalizes with the $60 \mathrm{~S}$ ribosome subunit and is required for its nuclear export (Hedges et al., 2006). Our results showed that NMD is regulated by vesicle-mediated trafficking, cytoskeleton proteins, extracellular matrix components and ubiquitin-mediated protein degradation, although the underlying mechanism for their role in NMD has yet to be elucidated. Thus, various biological processes could impinge on the regulation of the effectiveness of NMD in vivo.

\section{Role of translation in regulating RNAi}

A genome-wide RNAi screen using the RNAi sensor strain has identified four factors that are involved in protein synthesis, including translation initiation factor, ribosome subunit and factors involved in ribosome biogenesis, which, when inactivated, cause defects in RNAi (Kim et al., 2005). In this study, we identified 12 genes that are required for RNAi and six out of 12 genes identified in our screen are involved in translation, further demonstrating the link between translation and RNAi. Several lines of evidence suggest that RNAi may be linked with translation. The RISC complex directly interacts 
with ribosomal proteins L1, L11 and L7 and ribosomal biogenesis factor elF6 (Pham et al., 2004; Chendrimada et al., 2007). Furthermore, untranslated mRNAs are refractory to RNAi in Drosophila (Kennerdell et al., 2002). However, studies using the ferritin IRE-IRP system in mammalian cells indicate that active translation is not necessary for siRNA-mediated cleavage and RNAi is more sensitive in the absence of translation (Gu and Rossi, 2005). By examining the expression of a gfp reporter, whose expression is regulated by the RNAi pathway, we showed that ribosomal biogenesis components are required for RNAi, while various translation factors and tRNA synthetases may repress RNAi, indicating that different steps in protein synthesis may play a distinct role in regulating RNAi. Functional ribosomal subunits may be involved in recruiting the RISC complex to its target, while active translation prevents mRNA from being degraded by RNAi.

\section{Role of factors regulating the formation of $P$ bodies in NMD, RNAi and miRNA pathways}

$P$ bodies have been shown to be the place for NMD in yeast and mammalian cells and also for RNAi, miRNA-mediated gene silencing in mammalian cells (Jakymiw et al., 2005; Liu et al., 2005a; Parker and Sheth, 2007). However, recent studies indicate that mRNA decay, NMD and RNAi-mediated gene silencing are functional in Drosophila and mammalian cells lacking detectable microscopic $P$ bodies, suggesting that the formation of $\mathrm{P}$ bodies is a consequence of these processes (Chu and Rana, 2006; Eulalio et al., 2007). Here we revealed that a large number of genes whose inactivations enhance the formation of $P$ bodies are also involved in regulating NMD, RNAi- and miRNA-mediated gene silencing, providing a link between the formation of $P$ bodies and these RNA metabolism processes.

A genome-wide RNAi screen using the RNAi sensor strain identified 90 genes required for RNAi and silencing of the expression of scm::gfp in seam cells (Kim et al., 2005). Ten genes, ruvb-1, rha-2, ZK1127.5, T25G3.3, pro-2, E02H1.1, ima-3, F43G9.1, F26E4.4 and ZK1127.4, are also recovered in our screen, and when inactivated, lead to the increased number of $\mathrm{P}$ bodies. We identified nine additional genes that have not been recovered in the previous screen, indicating a certain degree of inter-experimental variation in high throughput genome-wide RNAi screen. The RNAi pathway is also required for silencing of repetitive sequence, including natural transposable elements and multicopies of transgene, in the $C$. elegans germline (Vastenhouw et al., 2003; Robert et al., 2005). The repetitive DNA can further silence the cognate endogenous germline-expressed genes, known as cosuppression, which is also regulated by RNAi (Robert et al., 2005). Genome-wide RNAi screens have identified 27 and 59 genes, and when silenced, cause transposition of Tc1 and defects in cosuppression in the $C$. elegans germline, respectively (Vastenhouw et al., 2003; Robert et al., 2005). Five out of 59 cde genes required for cosuppression and 4 out of 27 genes required for transposon silencing are included in our screen that regulate the formation of $\mathrm{P}$ bodies (Table 2 and Table S1). Taken together, 28 genes identified in our screen that enhance the formation of $P$ bodies are also required for RNAi-related processes.

miRNA-mediated gene silencing has also been shown to take place in $\mathrm{P}$ bodies (Liu et al., 2005b). A whole-genome RNAi screen has identified 213 candidate miRNA pathway genes whose inactivations enhance the bursting phenotype in let-7(mg279) mutants (Parry et al., 2007). 44 genes are further confirmed to be involved in specifying the temporal fate of seam cells regulated by let-7. These new miRNA pathway genes function downstream of let-7 expression and processing. 14 out of 213 candidates and 5 out of 44 confirmed let-7 pathway genes (ncbp-1, imb-4, B0285.1, let92 and F25B4.6) are included in our list that caused enhanced formation of $\mathrm{P}$ bodies (Table 2).

Components of NMD, siRNA and miRNA pathways are coexisted in $\mathrm{P}$ bodies and several components involved in mRNA degradation also play a role in RNAi and miRNA pathway (Rehwinkel et al., 2005). For example, XRN1 is recruited by both NMD and RNAi (Orban and Izaurralde, 2005). miRNA-mediated mRNA degradation requires both CCR4/NOT deadenylase and the DCP1/DCP2 complex (Behm-Ansmant and Izaurralde, 2006). Furthermore, the NMD factors SMG-2, $-5,-6$ are also required for persistence of RNAi, though not to initiate silencing (Domeier et al., 2000). In this study, we isolated a group of genes that are involved in both NMD and RNAi. We found that factors involved in ribosomal biogenesis and the CTP synthase/W06H3.3 are required for both RNAi and NMD (Table 2), indicating that there might be a common step shared by these two RNA degradation pathways. Seven genes that are involved in the RNAi pathway (dcr-1, pop-1, kin-10, H06/04.3, imb-4, C37A2.7 and eif-6), when inactivated, significantly enhance the bursting phenotype of let-7(mg279) (Table 2), indicating that although RNAi and miRNA pathways are largely molecularly distinct, there are few factors that are involved in both processes. Our study offers insights into the cellular mechanisms that regulate the formation of $\mathrm{P}$ bodies and also provides a framework for system-level understanding of NMD and RNAi in the context of the development of multicellular organisms.

\section{MATERIALS AND METHODS}

\section{Strains}

The following strains were used in this work: dcap-1(tm3163), dcap-2 (ok2023), bpEx10(bro-1::gfp; rol-6(+)), dpy-5(e61), dpy-5(e907), rrf-3 (ok629), eri-1(mg366), mut-2(r459), mut-7(pk204), wls51(scm::gfp), PTCx, ccEx7271(let-858p::gfp + pha-1), the RNAi sensor strain 
Table 2 List of identified genes that also function in RNAi-related and miRNA pathways

\begin{tabular}{|c|c|c|c|c|}
\hline$\overline{\mathrm{RNAi}}$ & miRNA & NMD & Functional description & H. sapiens \\
\hline$\overline{H 06 / 04.3^{1}}$ & $H 06104.3^{2}$ & H06I04.3 & SAM-dependent rRNA methyltransferase & FTSJ3 \\
\hline$i m b-4$ & $i m b-4^{2}$ & & Exportin-1/CRM1 & XPO1 \\
\hline \multirow[t]{4}{*}{ C $37 A 2.7^{3}$} & C37A2. $7^{2}$ & & Ribosomal protein, large subunit P2 & RPLP2 \\
\hline & $n c b p-1^{2}$ & $n c b p-1$ & Nuclear cap-binding protein, CBP80 & NCBP1 \\
\hline & $e m b-9^{2}$ & emb-9 & Collagen & \\
\hline & $R 06 C 7.5^{2}$ & $R 06 C 7.5$ & Adenylosuccinate lyase & \\
\hline ruvb-1 $1^{4}$ & & $r u v b-1$ & Recombination protein RUVB1 homolog & RUVBL1 \\
\hline rha- $2^{4}$ & & rha-2 & U3 snoRNP protein, DEAH-box RNA helicase & DHX37 \\
\hline T07A9. $8^{3}$ & & T07A9.8 & RNA methylase, pre-rRNA processing & KIAA0409 \\
\hline$T 25 G 3.3^{4}$ & & T25G3.3 & NMD3 homolog, 60S ribosomal biogenesis & NMD3 \\
\hline $\mathrm{E} 02 \mathrm{H} 1.1^{4}$ & & $E 02 H 1.1$ & rRNA adenine dimethylase & DIMT1L \\
\hline W06H3.3 & & W06H3.3 & CTP synthase & CTPS \\
\hline F26E4. $4^{4}$ & & F26E4.4 & Unknown function & \\
\hline Y77E11A. $7^{1}$ & & Y77E11A.7 & Unknown function & \\
\hline ZK1127.4 $4^{4}$ & & ZK1127.4 & Unknown function & \\
\hline$x r n-2^{3}$ & & & $5^{\prime}$ to $3^{\prime}$ exonuclease RAT1 & XRN2 \\
\hline ZK1127.5 & & & RNA 3' terminal phosphate cyclase & RCL1 \\
\hline pro- $2^{4}$ & & & Intranuclear ribosome transport & NOC2L \\
\hline ima-3 $3^{3}$ & & & Importin alpha family & KPNA4 \\
\hline$n p p-17^{1}$ & & & mRNA export protein Rae1 homolog & RAE1 \\
\hline$n p p-4^{1}$ & & & Nuclear pore complex, Nup98 & \\
\hline F43G9. $1^{4}$ & & & Isocitrate dehydrogenase a subunit & IDH3A \\
\hline \multirow[t]{9}{*}{ sas $-4^{3}$} & & & Spindle assembly factor & SASS6 \\
\hline & ZC123.3 $3^{2}$ & & Zinc finger homeodomain protein & \\
\hline & $r n p-4^{2}$ & & The EJC complex component $Y 14$ homolog & RBM8A \\
\hline & $n c b p-2^{2}$ & & Nuclear cap binding protein, CBP20 & \\
\hline & $Y 65 B 4 B R \cdot 5^{2}$ & & NAC component & NACA \\
\hline & $u b c-9^{2}$ & & SUMO conjugating enzyme & \\
\hline & $B 0285.1^{2}$ & & $c d c 2$-related protein kinase & CDC2L5 \\
\hline & let-92 ${ }^{2}$ & & Phosphatase $2 \mathrm{~A}$, catalytic subunit & PPP2CB \\
\hline & F25B4.6 & & HMG-CoA synthase & HMGCS2 \\
\hline
\end{tabular}

\footnotetext{
${ }^{1}$ Identified as factors required for transposon silencing by Vastenhouw et al. (2003).

2 Identified as miRNA related genes by Parry et al. (2007).

${ }^{3}$ Identified as cde (cosuppression defective) genes by Robert et al. (2005).

${ }^{4}$ Identified as RNAi factors by Kim et al. (2005).
}

GR1401. bp/s37(dcap-1::rfp + rol-6(su1006)), bp/s70(gfp::bro-1 $(P T C)+$ rol-6(su1006)), bpls88(tia-1::gfp + dcap-1::rfp + rol-6 (su1006)) and bp/s90[(tia-1::gfp + rol-6(su1006)].

\section{Reporters}

To construct dcap-1 reporter (bp/s37), the genomic DNA of dcap-1, containing the promoter, exon and intron sequence (cosmid Y55F3AM: nt 77,354-73,583), was amplified and inserted at the sal-1 and BamHI sites of the plasmid pPD95.79 (RFP). dcap-1::rfp DNA $(1 \mathrm{ng} / \mu \mathrm{L})$ was coinjected with $p R F 4(r o l-6)$ into N2 wild type animals. Construction of bro-1::gfp report was previously described
(Xia et al., 2007). To construct gfp::bro-1(PTC) reporter (bp/s70), a premature termination codon was introduced at the 91st codon in BRO-1 using QuikChange mutagenesis (Stratagene). tia-1::gfp reporter (bp/s90) was constructed by inserting the promoter sequence of tia-1 (C18A3: complement, nt 15,368-13,381) into ppD95.79 at the HindIII and Pstl sites. Subsequently, the coding region and 3' UTR of tia-1 (C18A3: complement, nt 13,380-10,410) was inserted at the C-terminus of gfp at the Nhel and Sacll sites. tia$1:: g f p(20 \mathrm{ng} / \mu \mathrm{L})$ was co-injected with dcap-1::rfp $(10 \mathrm{ng} / \mu \mathrm{L})$ and pRF4(rol-6) into N2 animals. Stable integrated transgenic lines for each reporter were obtained after $\mathrm{Y}$-ray irradiation and were outcrossed at least twice. 


\section{Preparation and induction of RNAi bacterial clones}

The RNAi feeding library is commercially available from Geneservice. The library contains bacterial clones expressing dsRNA designed to individually inactivate 16,644 genes (targeting about $87 \%$ of the genome). RNAi bacterial clones were grown on LB-agar plates supplemented with $100 \mathrm{mg} / \mathrm{mL}$ ampicillin and $30 \mathrm{mg} / \mathrm{mL}$ tetracycline, and then inoculated into LB medium containing $50 \mathrm{mg} / \mathrm{mL}$ ampicillin and cultured for $6 \mathrm{~h}$ at $37^{\circ} \mathrm{C}$. $300 \mu \mathrm{L}$ of each bacterial culture was dispensed onto $10 \mathrm{~cm} \mathrm{NGM} \mathrm{agar} \mathrm{plates} \mathrm{containing} 5 \mathrm{mmol} / \mathrm{L}$ IPTG (Sigma). dsRNA transcription was induced for $12-16 \mathrm{~h}$ at $25^{\circ} \mathrm{C}$.

\section{Identifying RNAi screen with enhanced formation of $\mathrm{P}$ bodies}

Synchronized L1 dcap-1::rfp; rrf-3 animals were plated onto RNAi feeding plates with approximately 10-15 worms per plate and were grown at $20^{\circ} \mathrm{C}$ for 5 days. The $\mathrm{F} 1$ progeny or arrested larvae or sterile adults were examined for reporter expression. The expression level of the reporter was classified into five classes, N, wl, I, II, III (N: no change in the expression of the reporter compared to animals fed on control RNAi clone containing the empty L4440 vector; wl: RFP expression is faintly brighter than baseline in the whole animal in most worms; I: slightly brighter in most worms; II: brighter in the whole animal or specific tissues in most animals. III: the expression of RFP is very bright in the whole animal or specific tissues in most animals. All RNAi clones that enhanced the formation of $P$ bodies were subjected to three more rounds of testing. 360 RNAi clones that enhanced the formation of $P$ bodies in at least three separate tests were selected for further analysis.

To examine the expression of let-858p::gfp, synchronized L1 let$858 p:: g f p$ animals were plated onto RNAi feeding plates with approximately 10 worms per plate and were grown at $20^{\circ} \mathrm{C}$ for 5 days. The F1 progeny or arrested larvae or sterile adults were examined for reporter expression. Of the 360 identified RNAi clones, 136 RNAi clones caused obvious enhanced expression of let-858p:: gfp.

\section{Identification of genes with a role in regulation of NMD}

The 216 RNAi clones that enhanced the formation of $\mathrm{P}$ bodies in the whole animal were screened for their roles in NMD using gfp::bro-1 $(P T C)$ reporter. Five to seven L4 animals carrying a gfp::bro-1(PTC) transgene were plated onto RNAi feeding plates and were grown at $21^{\circ} \mathrm{C}$ for 3 days. The F1 L3 or L4 larvae were examined for reporter expression. Expression of gfp::bro-1(PTC) was not detected in late larvae fed on control RNAi clone $(n>100)$. The ratio of animals expressing gfp::bro-1(PTC) was derived from three separate experiments (at least 30 animals were examined for each RNAi clone). The RNAi clones that caused more than $25 \%$ of animals expressing gfp were selected for further analysis.

The identified 83 RNAi clones were further tested for their effects on the expression of PTCx reporter. About ten synchronized L1 animals carrying the PTCx reporter were plated onto RNAi feeding plates and were grown at $20^{\circ} \mathrm{C}$ for 5 days. The $\mathrm{F} 1$ progeny or arrested larvae or sterile adults were examined for the expression of the reporter.

The identified RNAi clones with increased expression level of $g f p:$ : bro-1(PTC) were further analyzed for suppression of $d p y-5(e 61)$. Five to seven L4 dpy-5(e61); rrf-3 mutant animals were seeded onto RNAi feeding plates and were grown at $20^{\circ} \mathrm{C}$ for 4 days, and the deletion mutant $d p y$-5(e907) was applied parallel as control. The body size of F1 adult animals was measured under a microscope. The experiments were repeated three times.

\section{Identification of mutants that enhance the formation of TIA-1 bodies}

The 224 identified RNAi clones that enhanced the formation of $\mathrm{P}$ bodies in the whole animal or in specific tissues were further screened for the expression of tia-1. The RNAi sensitive strain eri-1 carrying the tia-1::gfp and dcap-1::rfp reporters were used for screen. The expression level of the reporter was classified into five levels as described above for the formation of $\mathrm{P}$ bodies.

\section{Identification of genes with a role in regulation of RNAi}

The 216 RNAi clones were screened for increasing the expression of scm::gfp in rrf-3 mutants. The GFP level of worms fed on control RNAi clone was set as baseline. The experiments were repeated six times and the increased expression level of the reporter was scored as + , ++ , and +++ (strongest). The 12 identified genes in the above assay were further tested for the expression of $s c m:: g f p$ in the RNAi sensor strain (Kim et al., 2005). In brief, expression of scm::gfp in the RNAi sensor strain was silenced by a transgene that simultaneously expresses the sense and antisense gfp RNA. However, scm::gfp was not uniformly silenced in this strain and some animals still showed strong expression. Only the RNAi clones that led to significantly elevated expression of $s c m:: g f p$ in most of the animals were selected as positive clones. We also screened the 216 RNAi clones to identify mutants that reduced the expression of scm::gfp. The identified RNAi clones with reduced expression level of $g f p$ were further examined for the expression of scm::gfp in mut-7 or mut-2 mutants; both genes have been shown to be involved in RNAi. By determining the expression pattern of the germ granule specific marker pgl-1::gfp, we found that $P$ granules remained to be restricted in germline in all identified mutants. Therefore, enhanced RNAi in the identified mutants was not due to ectopic expression of germ granules in somatic tissues, as in lin-35 Rb pathway mutants (Wang et al., 2005).

\section{Sequencing of RNAi clones}

The identified RNAi clones were sequenced using primers located in the pL4440 vector (forward primer: 5'-CCTGGCTTATCGAAATTAATAC-3'; reverse primer: 5'-CTCACTATAGGGCGAATTGG-3').

\section{RT-PCR}

The expression level of gfp::bro-1(PTC) (forward primer: 5'-CACTGGAGTTGTCCCAATTCT-3'; reverse primer: 5'-GTATAGTTCATCCATGCCATG-3') was measured by RT-PCR. RT-PCR was performed using SuperScript ${ }^{\mathrm{TM}}$ One-Step RT-PCR with PlatinumTaq kit (Invitrogen) according to the manufacturer's manual.

\section{ACKNOWLEDGEMENTS}

We are grateful to Dr. Kim John for the RNAi sensor strain, to Dr. Javier F. Cáceres for PTCx report and to Dr. Isabel Hanson for 
critically evaluating the manuscript. This work was supported by the National High Technology Research and Development Program of China (863 Program) (Grant No. 2005AA210910).

Supplementary material is available in the online version of this article at http://dx.doi.org/10.1007/s13238-011-1119-x and is accessible for authorized users.

\section{ABBREVIATIONS}

EJC, the exon junction complex; NMD, nonsense-mediated RNA decay; PTC, premature termination codon; RNP, ribonucleoprotein; SGs, stress granules

\section{REFERENCES}

Anderson, P., and Kedersha, N. (2006). RNA granules. J Cell Biol 172, 803-808.

Basu, U., Si, K., Warner, J.R., and Maitra, U. (2001). The Saccharomyces cerevisiae TIF6 gene encoding translation initiation factor 6 is required for 605 ribosomal subunit biogenesis. Mol Cell Biol 21, 1453-1462.

Behm-Ansmant, I., and Izaurralde, E. (2006). Quality control of gene expression: a stepwise assembly pathway for the surveillance complex that triggers nonsense-mediated mRNA decay. Genes Dev 20, 391-398.

Brengues, M., Teixeira, D., and Parker, R. (2005). Movement of eukaryotic mRNAs between polysomes and cytoplasmic processing bodies. Science 310, 486-489.

Bruno, I., and Wilkinson, M.F. (2006). P-bodies react to stress and nonsense. Cell 125, 1036-1038.

Chen, C.C., Simard, M.J., Tabara, H., Brownell, D.R., McCollough, J. A., and Mello, C.C. (2005). A member of the polymerase beta nucleotidyltransferase superfamily is required for RNA interference in C. elegans. Curr Biol 15, 378-383.

Chendrimada, T.P., Finn, K.J., Ji, X., Baillat, D., Gregory, R.I., Liebhaber, S.A., Pasquinelli, A.E., and Shiekhattar, R. (2007). MicroRNA silencing through RISC recruitment of elF6. Nature 447, 823-828.

Chu, C.Y., and Rana, T.M. (2006). Translation repression in human cells by microRNA-induced gene silencing requires RCK/p54. PLoS Biol 4, e210.

Cougot, N., Babajko, S., and Séraphin, B. (2004). Cytoplasmic foci are sites of mRNA decay in human cells. J Cell Biol 165, 31-40.

Cui, Y., González, C.I., Kinzy, T.G., Dinman, J.D., and Peltz, S.W. (1999). Mutations in the MOF2/SUl1 gene affect both translation and nonsense-mediated mRNA decay. RNA 5, 794-804.

Domeier, M.E., Morse, D.P., Knight, S.W., Portereiko, M., Bass, B.L., and Mango, S.E. (2000). A link between RNA interference and nonsense-mediated decay in Caenorhabditis elegans. Science 289, 1928-1931.

Eulalio, A., Behm-Ansmant, I., Schweizer, D., and Izaurralde, E. (2007). P-body formation is a consequence, not the cause, of RNAmediated gene silencing. Mol Cell Biol 27, 3970-3981.

Gu, S., and Rossi, J.J. (2005). Uncoupling of RNAi from active translation in mammalian cells. RNA 11, 38-44.

Halawani, D., and Latterich, M. (2006). p97: The cell's molecular purgatory? Mol Cell 22, 713-717.

He, F., and Jacobson, A. (1995). Identification of a novel component of the nonsense-mediated mRNA decay pathway by use of an interacting protein screen. Genes Dev 9, 437-454.

Hedges, J., Chen, Y.I., West, M., Bussiere, C., and Johnson, A.W. (2006). Mapping the functional domains of yeast NMD3, the nuclear export adapter for the $60 \mathrm{~S}$ ribosomal subunit. J Biol Chem 281, 36579-36587.

Hodgkin, J., Papp, A., Pulak, R., Ambros, V., and Anderson, P. (1989). A new kind of informational suppression in the nematode Caenorhabditis elegans. Genetics 123, 301-313.

Hosoda, N., Kim, Y.K., Lejeune, F., and Maquat, L.E. (2005). CBP80 promotes interaction of Upf1 with Upf2 during nonsense-mediated mRNA decay in mammalian cells. Nat Struct Mol Biol 12, 893-901.

Houseley, J., LaCava, J., and Tollervey, D. (2006). RNA-quality control by the exosome. Nat Rev Mol Cell Biol 7, 529-539.

Isken, O., and Maquat, L.E. (2007). Quality control of eukaryotic mRNA: safeguarding cells from abnormal mRNA function. Genes Dev 21, 1833-1856.

Jakymiw, A., Lian, S., Eystathioy, T., Li, S., Satoh, M., Hamel, J.C., Fritzler, M.J., and Chan, E.K. (2005). Disruption of GW bodies impairs mammalian RNA interference. Nat Cell Biol 7, 1267-1274.

Johnson, A.W. (1997). Rat1p and Xrn1p are functionally interchangeable exoribonucleases that are restricted to and required in the nucleus and cytoplasm, respectively. Mol Cell Biol 17, 6122-6130.

Kedersha, N., Stoecklin, G., Ayodele, M., Yacono, P., LykkeAndersen, J., Fritzler, M.J., Scheuner, D., Kaufman, R.J., Golan, D.E., and Anderson, P. (2005). Stress granules and processing bodies are dynamically linked sites of mRNP remodeling. J Cell Biol 169, 871-884.

Kedersha, N.L., Gupta, M., Li, W., Miller, I., and Anderson, P. (1999). RNA-binding proteins TIA-1 and TIAR link the phosphorylation of elF-2 alpha to the assembly of mammalian stress granules. J Cell Biol 147, 1431-1442.

Kennedy, S., Wang, D., and Ruvkun, G. (2004). A conserved siRNA-degrading RNase negatively regulates RNA interference in C. elegans. Nature 427, 645-649.

Kennerdell, J.R., Yamaguchi, S., and Carthew, R.W. (2002). RNAi is activated during Drosophila oocyte maturation in a manner dependent on aubergine and spindle-E. Genes Dev 16, 1884-1889.

Ketting, R.F., Haverkamp, T.H., van Luenen, H.G., and Plasterk, R.H. (1999). Mut-7 of C. elegans, required for transposon silencing and RNA interference, is a homolog of Werner syndrome helicase and RNaseD. Cell 99, 133-141.

Kim, J.K., Gabel, H.W., Kamath, R.S., Tewari, M., Pasquinelli, A., Rual, J.F., Kennedy, S., Dybbs, M., Bertin, N., Kaplan, J.M., et al. (2005). Functional genomic analysis of RNA interference in $C$. elegans. Science 308, 1164-1167.

Kimball, S.R., Horetsky, R.L., Ron, D., Jefferson, L.S., and Harding, H.P. (2003). Mammalian stress granules represent sites of accumulation of stalled translation initiation complexes. Am J Physiol Cell Physiol 284, C273-C284.

Liu, H.Y., Chiang, Y.C., Pan, J., Chen, J., Salvadore, C., Audino, D.C., Badarinarayana, V., Palaniswamy, V., Anderson, B., and Denis, C. L. (2001). Characterization of CAF4 and CAF16 reveals a functional connection between the CCR4-NOT complex and a subset of SRB proteins of the RNA polymerase II holoenzyme. J Biol Chem 276, 7541-7548.

Liu, J., Rivas, F.V., Wohlschlegel, J., Yates, J.R. 3rd, Parker, R., and 
Hannon, G.J. (2005a). A role for the P-body component GW182 in microRNA function. Nat Cell Biol 7, 1261-1266.

Liu, J., Valencia-Sanchez, M.A., Hannon, G.J., and Parker, R. (2005b). MicroRNA-dependent localization of targeted mRNAs to mammalian P-bodies. Nat Cell Biol 7, 719-723.

Longman, D., Plasterk, R.H., Johnstone, I.L., and Cáceres, J.F. (2007). Mechanistic insights and identification of two novel factors in the C. elegans NMD pathway. Genes Dev 21, 1075-1085.

Lotan, R., Bar-On, V.G., Harel-Sharvit, L., Duek, L., Melamed, D., and Choder, M. (2005). The RNA polymerase II subunit Rpb4p mediates decay of a specific class of mRNAs. Genes Dev 19, 3004-3016.

Mango, S.E. (2001). Stop making nonSense: the C. elegans smg genes. Trends Genet 17, 646-653.

Maquat, L.E. (2004). Nonsense-mediated mRNA decay: splicing, translation and mRNP dynamics. Nat Rev Mol Cell Biol 5, 89-99.

Orban, T.I., and Izaurralde, E. (2005). Decay of mRNAs targeted by RISC requires XRN1, the Ski complex, and the exosome. RNA 11, 459-469.

Parker, R., and Sheth, U. (2007). P bodies and the control of mRNA translation and degradation. Mol Cell 25, 635-646.

Parry, D.H., Xu, J., and Ruvkun, G. (2007). A whole-genome RNAi Screen for C. elegans miRNA pathway genes. Curr Biol 17, 2013-2022.

Pham, J.W., Pellino, J.L., Lee, Y.S., Carthew, R.W., and Sontheimer, E.J. (2004). A Dicer-2-dependent 80s complex cleaves targeted mRNAs during RNAi in Drosophila. Cell 117, 83-94.

Rehwinkel, J., Behm-Ansmant, I., Gatfield, D., and Izaurralde, E. (2005). A crucial role for GW182 and the DCP1:DCP2 decapping complex in miRNA-mediated gene silencing. RNA 11, 1640-1647.

Robert, V.J., Sijen, T., van Wolfswinkel, J., and Plasterk, R.H. (2005). Chromatin and RNAi factors protect the $C$. elegans germline against repetitive sequences. Genes Dev 19, 782-787.

Sen, G.L., and Blau, H.M. (2005). Argonaute 2/RISC resides in sites of mammalian mRNA decay known as cytoplasmic bodies. Nat Cell Biol 7, 633-636.

Sheth, U., and Parker, R. (2003). Decapping and decay of messenger RNA occur in cytoplasmic processing bodies. Science 300 , 805-808.

Sheth, U., and Parker, R. (2006). Targeting of aberrant mRNAs to cytoplasmic processing bodies. Cell 125, 1095-1109.

Simmer, F., Tijsterman, M., Parrish, S., Koushika, S.P., Nonet, M.L., Fire, A., Ahringer, J., and Plasterk, R.H. (2002). Loss of the putative RNA-directed RNA polymerase RRF-3 makes $C$. elegans hypersensitive to RNAi. Curr Biol 12, 1317-1319.

Teixeira, D., and Parker, R. (2007). Analysis of P-body assembly in Saccharomyces cerevisiae. Mol Biol Cell 18, 2274-2287.

Vastenhouw, N.L., Fischer, S.E., Robert, V.J., Thijssen, K.L., Fraser, A.G., Kamath, R.S., Ahringer, J., and Plasterk, R.H. (2003). A genome-wide screen identifies 27 genes involved in transposon silencing in C. elegans. Curr Biol 13, 1311-1316.

Wang, D., Kennedy, S., Conte, D. Jr, Kim, J.K., Gabel, H.W., Kamath, R.S., Mello, C.C., and Ruvkun, G. (2005). Somatic misexpression of germline $P$ granules and enhanced RNA interference in retinoblastoma pathway mutants. Nature 436, 593-597.

Xia, D., Zhang, Y., Huang, X., Sun, Y., and Zhang, H. (2007). The C. elegans CBFbeta homolog, BRO-1, regulates the proliferation, differentiation and specification of the stem cell-like seam cell lineages. Dev Biol 309, 259-272.

Zhong, W., and Sternberg, P.W. (2006). Genome-wide prediction of $C$. elegans genetic interactions. Science 311, 1481-1484.

Zuk, D., Belk, J.P., and Jacobson, A. (1999). Temperature-sensitive mutations in the Saccharomyces cerevisiae MRT4, GRC5, SLA2 and THS1 genes result in defects in mRNA turnover. Genetics 153 , $35-47$. 